Pacific

Journal of

Mathematics

ANALYTIC FLOWS ON THE UNIT DISK: ANGULAR DERIVATIVES AND BOUNDARY FIXED POINTS

Manuel D. Contreras AND SANTiago DíaZ-Madrigal 


\title{
ANALYTIC FLOWS ON THE UNIT DISK: ANGULAR DERIVATIVES AND BOUNDARY FIXED POINTS
}

\author{
Manuel D. Contreras and SAntiago DíaZ-Madrigal
}

\begin{abstract}
We use the concept of angular derivative and the hyperbolic metric in the unit disk $\mathbb{D}$, to study the dynamical aspects of the equilibrium points belonging to $\partial \mathbb{D}$ of some complex-analytic dynamical systems on $\mathbb{D}$. Our results show a deep connection between the dynamical properties of those equilibrium points and the geometry of certain simply connected domains of $\mathbb{C}$. As a consequence, and in the context of semigroups of analytic functions, we give some geometric insight to a well-known inequality of Cowen and Pommerenke about the angular derivative of an analytic function.
\end{abstract}

\section{Introduction}

A (one-parameter) semigroup of analytic functions is any continuous homomorphism $\Phi: t \mapsto \Phi(t)=\varphi_{t}$ from the additive semigroup of nonnegative real numbers into the composition semigroup of all analytic functions which map $\mathbb{D}$ into $\mathbb{D}$. That is, $\Phi$ satisfies the following three conditions:

(a) $\varphi_{0}$ is the identity in $\mathbb{D}$,

(b) $\varphi_{t+s}=\varphi_{t} \circ \varphi_{s}$, for all $t, s \geq 0$,

(c) $\varphi_{t}(z)$ tends to $z$ as $t$ tends to 0 , uniformly on compact subsets of $\mathbb{D}$.

It is well-known that condition (c) can be replaced by

(c') For every $z \in \mathbb{D}, \lim _{t \rightarrow 0} \varphi_{t}(z)=z$.

Semigroups of this type appear in many areas of analysis, such as the theory of composition operators, the theory of Markov stochastic processes, optimization theory and the theory of planar vector fields. In this paper, we are interested in this last aspect, which we discuss in detail for completeness.

MSC2000: primary 30C20, 30D05, 37F99; secondary 30F45, 37E35.

Keywords: angular derivative, fixed points, planar vector fields, semigroups of analytic functions. This research has been partially supported by the Ministerio de Ciencia y Tecnología (projects BFM2000-1062 and BFM2003-07294-C02-02) and by La Consejería de Educación y Ciencia de la Junta de Andalucía. 
Given a semigroup $\Phi=\left(\varphi_{t}\right)$, it can be proved (see [Shoikhet 2001; Berkson and Porta 1978]) that there exists a unique analytic function $G: \mathbb{D} \rightarrow \mathbb{C}$ such that, for each $z \in \mathbb{D}$, the trajectory

$$
\gamma_{z}:[0,+\infty) \rightarrow \mathbb{R}^{2}, \quad t \mapsto \gamma_{z}(t):=\varphi_{t}(z)
$$

is the solution of the Cauchy problem

$$
\left\{\begin{array}{l}
\dot{x}=f(x, y), \quad \dot{y}=g(x, y), \\
(x(0), y(0))=z
\end{array}\right.
$$

where $f=\operatorname{Re} G$ and $g=\operatorname{Im} G$. Usually, this planar dynamical system is written in the form $\dot{w}=G(w)$, that is, as a complex-analytic dynamical system in $\mathbb{D}$. We call $G$ the vector field, and $\dot{w}=G(w)$ the dynamical system, associated with $\Phi$. In operator theory, $G$ is also known as the infinitesimal generator of $\Phi$. There is a very nice representation, due to Berkson and Porta [1978], of those analytic functions on the disk which are generated in this way:

An analytic function $G: \mathbb{D} \rightarrow C$ is the vector field of a semigroup of analytic functions $\Phi$ if and only if there is a point $b \in \overline{\mathbb{D}}$ and an analytic function $p: \mathbb{D} \rightarrow \mathbb{C}$ with $\operatorname{Re} p \geq 0$ such that

$$
G(z)=(b-z)(1-\bar{b} z) p(z), \quad z \in \mathbb{D} .
$$

Moreover, such a representation is unique. The point $b$ is called the Denjoy-Wolff point (DW-point) of $\Phi$ and $p$ is called the Carathéodory function associated to $\Phi$.

Looking at this representation, we see that, whenever $b \in \mathbb{D}$, the associated dynamical system has a critical point. However, $G$ never vanishes if $b \in \partial \mathbb{D}$. Moreover, it is well known that complex-analytic dynamical systems in simply connected domains cannot have limit cycles [Needham and King 1994], so it is natural to wonder about the "elements" which govern (and how they do it) the dynamics of this system without critical points and without limit cycles. In the rest of this section, we will see that the key for understanding this dynamics is the study of some "critical points" belonging to $\partial \mathbb{D}$. Since, in our setting, $G$ and the functions $\left(\varphi_{t}\right)$ are only defined in $\mathbb{D}$, that comment requires a clarification and this leads us to recall some concepts from complex function theory [Pommerenke 1975; 1992; Shapiro 1993].

Let $\varphi: \mathbb{D} \rightarrow \mathbb{C}$ be an analytic function and take $a \in \partial \mathbb{D}$. We say that $L \in \mathbb{C}_{\infty}$ is the angular limit of $\varphi$ in $a$ when $z$ tends to $a$ if, for every $\alpha \in(0, \pi / 2)$,

$$
\lim _{\substack{z \in S(a, \alpha) \\ z \rightarrow a}} \varphi(z)=L
$$

where $S(a, \alpha)$ denotes the Stolz angle with center at $a$ and opening $\alpha$. The number $L$ is commonly denoted by $\angle \lim _{z \rightarrow a} \varphi(z)$. When $\angle \lim _{z \rightarrow a} \varphi(z)=a$, the point $a$ 
is called a boundary fixed point of $\varphi$. If we suppose additionally that $\varphi(\mathbb{D}) \subset \mathbb{D}$, the angular limit

$$
\angle \lim _{z \rightarrow a} \frac{\varphi(z)-a}{z-a}
$$

always exists and belongs to $(0,+\infty) \cup\{\infty\}$. This value is denoted by $\varphi^{\prime}(a)$ and we refer to it as the multiplier of $\varphi$ in $a$. When $\varphi^{\prime}(a) \in(0,+\infty)$, the limit $\angle \lim _{z \rightarrow a} \varphi^{\prime}(z)$ exists and is equal to $\varphi^{\prime}(a)$. Following the more or less standard usage in iteration theory, we will say that $a$ is attractive if $\varphi^{\prime}(a) \in(0,1]$; repulsive if $\varphi^{\prime}(a) \in(1,+\infty)$ and superrepulsive if $\varphi^{\prime}(a)=\infty$. These definitions are consistent with the intuitive geometric meaning of a repulsive or attractive point, thanks to the celebrated Denjoy-Wolff Theorem [Shapiro 1993, Section 5.1].

Now, we come back to the semigroup $\Phi=\left(\varphi_{t}\right)$ and the associated vector field $G$ with DW-point $b \in \partial \mathbb{D}$. A point $a \in \partial \mathbb{D}$ is a boundary fixed point of $\Phi$ if $a$ is a boundary fixed point for each $\varphi_{t}$, where $t \geq 0$. It is a nontrivial fact due to Cowen [1981, Theorem 5.2] that "for each $\varphi_{t}$ " can be replaced by "for some $\varphi_{t}$ ". Moreover, the DW-point $b$ is a boundary fixed point of $\Phi$ and, indeed, the DenjoyWolff Theorem admits the following version for semigroups (see [Berkson and Porta 1978; Siskakis 1985]):

The point $b$ is the unique boundary fixed point of $\Phi$ with $\varphi_{t}^{\prime}(b) \leq 1$ for some (hence for all) $t>0$. Moreover, for every $z \in \mathbb{D}$, we have $\lim _{t \rightarrow+\infty} \varphi_{t}(z)=b$.

Combining the results of [Siskakis 1985] and [Cowen 1981], it is possible to deduce that the dynamical character of the multipliers of all the functions $\varphi_{t}$ is the same. That is, a point $\xi \in \partial \mathbb{D}$ is attractive (or repulsive, or superrepulsive) for some $t$ if and only if the same happens for all $t$.

Our study of the system $\dot{w}=G(w)$ will be carried out by considering a model flow where the trajectories become straight lines but they fill in a more involved planar domain $\Omega$. In other words, our results will explain the dynamics of $\dot{w}=$ $G(w)$ in terms of the geometry of $\Omega$. This domain $\Omega$ is constructed using the theory of univalent functions [Berkson and Porta 1978; Heins 1981; Siskakis 1985]: there is a unique univalent function $h: \mathbb{D} \rightarrow \mathbb{C}$ with $h(0)=0$ whose image $\Omega:=h(\mathbb{D})$ satisfies,

$$
\Omega+t \subset \Omega \quad \text { for each } t>0
$$

and such that

$$
\varphi_{t}(z)=b h^{-1}(h(\bar{b} z)+t) \quad \text { for } t \geq 0, z \in \mathbb{D} .
$$

We call $\Omega:=h(\mathbb{D}) \subset \mathbb{C}$ the associated planar domain of $\Phi$. Clearly, if we fix $z \in \mathbb{D}$ and consider the trajectory $t \rightarrow \gamma_{z}(t)=\varphi_{t}(z)$ with respect to the vector field $G$, the corresponding trajectory in the model flow is $t \rightarrow h(z)+t$.

Our dynamical approach to $\dot{w}=G(w)$ deals with the following topics: types of trajectories, their $\omega$-limits and $\alpha$-limits and their relationship with the boundary 
fixed points; the multipliers of these points and their dynamic meaning; the slope of the trajectories along which they arrive at such a fixed point. We recall that a point $\xi \in \mathbb{C}_{\infty}$ is called an $\omega$-limit point of a curve $\Gamma:\left(s_{1}, s_{2}\right) \rightarrow \mathbb{C}\left(-\infty \leq s_{1}<s_{2} \leq+\infty\right)$ if there exists a strictly increasing sequence $\left(t_{n}\right) \subset\left(s_{1}, s_{2}\right)$ converging to $s_{2}$ such that $\Gamma\left(t_{n}\right) \rightarrow \xi$. The set of all $\omega$-limit points of $\Gamma$ is called the $\omega$-limit set and denoted by $\omega(\Gamma)$. The definitions of $\alpha$-limit and $\alpha(\Gamma)$ can be given in a similar way but now the role of $s_{2}$ is played by the point $s_{1}$.

From the point of view of $\omega$-limits, and in our context, the result of Berkson and Porta cited above reads as follows:

Let $\Phi$ be a semigroup of analytic functions with boundary DW-point $b$, planar domain $\Omega$ and vector field $G$. Moreover, let $\gamma_{z}$ be a trajectory of the vector field $G$. Then $\gamma_{z}$ is defined for every $t \geq 0, \lim _{t \rightarrow+\infty} \gamma_{z}(t)=b$ and its largest possible interval of definition is $(T,+\infty)$ with $-\infty \leq T<0$.

The article contains four sections after this introduction. In Section 2 we state the main results of the paper. With the aim of making the paper more readable, we have grouped in Section 3 some facts about the hyperbolic distance on the unit disk. In Section 4 we obtain new results about the relationship among boundary fixed points, nontangential convergence and angular derivate. Some of these results may have some interest of their own, but here we think of them as necessary ingredients for the proof of the main results. In Section 5 we give the proofs of all the results stated in Section 2.

\section{Statement of the main results}

For the analysis of the multipliers of $\varphi_{t}$ at the DW-point $b$, we introduce a geometric quantity associated to the planar domain $\Omega$. Namely, let us denote by $v(\Omega)$ the supremum of all positive numbers $\beta$ such that there is $c \in \mathbb{C}$ with

$$
\left\{c+t i:-\frac{1}{2} \beta<t<\frac{1}{2} \beta\right\} \subset \Omega .
$$

We point out that this is a well-defined concept since $\Omega$ is open and nonvoid. Clearly, $v(\Omega) \in(0,+\infty]$. It is an exercise to show that, whenever $v(\Omega)<+\infty$, the number $v(\Omega)$ coincides with the infimum of all positive $\beta$ such that, there is $c \in \mathbb{R}$ with

$$
\Omega \subseteq\left\{z \in \mathbb{C}: c-\frac{1}{2} \beta<\operatorname{Im} z<c+\frac{1}{2} \beta\right\} .
$$

Bearing in mind the property $\Omega+t \subset \Omega$, we see that $\nu(\Omega)$ represents the "narrowest" width of an open strip parallel to the real axis and completely enclosing $\Omega$.

The following theorem explains the relation between $v(\Omega)$ and the multipliers of $\varphi_{t}$ in the DW-point $b$, when $v(\Omega)<\infty$. 
Theorem 2.1. Let $\Phi=\left(\varphi_{t}\right)$ be a semigroup of analytic functions with boundary $D W$-point $b$ and planar domain $\Omega$. The following statements are equivalent.

(1) $v(\Omega)<\infty$.

(2) For all $t>0$, we have $\varphi_{t}^{\prime}(b)<1$.

(3) There is $t>0$ such that $\varphi_{t}^{\prime}(b)<1$.

Moreover, when $v(\Omega)<\infty$, it follows that $\varphi_{t}^{\prime}(b)=\exp \left(-\frac{\pi}{v(\Omega)} t\right)$ for all $t \geq 0$.

From Theorem 2.1 and the fact that $\varphi_{t}^{\prime}(b) \leq 1$ for some (and therefore all) $t>0$, we readily deduce:

Corollary 2.2. Let $\Phi=\left(\varphi_{t}\right)$ be a semigroup of analytic functions with boundary $D W$-point $b$ and planar domain $\Omega$. The following statements are equivalent.

(1) $v(\Omega)=\infty$.

(2) For all $t>0$, we have $\varphi_{t}^{\prime}(b)=1$.

(3) There is $t>0$ such that $\varphi_{t}^{\prime}(b)=1$.

The relation between boundary fixed points and the backward evolution of the trajectories is expressed in the next proposition.

Proposition 2.3. Let $\Phi=\left(\varphi_{t}\right)$ be a semigroup of analytic functions with boundary $D W$-point $b$, planar domain $h(\mathbb{D})=\Omega$ and vector field $G$. Moreover, let $\gamma_{z}$ be a trajectory of the vector field $G$ with the maximum possible interval of definition $(T,+\infty)$ being $-\infty \leq T<0$.

(1) If $-\infty<T$, the limit

$$
a:=\lim _{s \rightarrow T} \gamma_{z}(s) \in \partial \mathbb{D},
$$

exists, $a$ is not a boundary fixed point of $\Phi$, and $h(z)+T \in \partial \Omega$.

(2) If $T=-\infty$, the limit

$$
a:=\lim _{s \rightarrow-\infty} \gamma_{z}(s) \in \partial \mathbb{D}
$$

exists and $a$ is a boundary fixed point of $\Phi$.

In our analysis of backward trajectories, a crucial step will be to develop tools in order to detect when different trajectories go to the same boundary fixed point. For this, we introduce the planar subset

$$
V(\Omega):=\operatorname{int}\left(\bigcap_{t \geq 0}(\Omega+t)\right) .
$$

We will check that $V(\Omega)+t=V(\Omega)$, for every $t \in \mathbb{R}$, so we call it the invariant set associated to $\Omega$. It is worth mentioning that $V(\Omega)$ can be empty. But, if $V(\Omega)$ 
is nonvoid and since it is obviously open, we can write $V(\Omega)$ as the union of the countable (finite or infinite) family of its different connected components. These components will be denoted by $V_{j}(\Omega)(j \in J)$ and, depending on the case, $J=\mathbb{N}$ or $J=\{1, \ldots, n\}$ for some $n \in \mathbb{N}$.

It can be proved that every component $V_{j}(\Omega)$ of the invariant set $V(\Omega)$ is an open strip or a half-plane parallel to the real axis in both cases.

Theorem 2.4. Let $\Phi=\left(\varphi_{t}\right)$ be a semigroup of analytic functions with boundary $D W$-point $b$ and planar domain $\Omega=h(\mathbb{D})$.

(1) If a component $V_{j}(\Omega)$ of $V(\Omega)$ is a half-plane, then for every $z \in h^{-1}\left(V_{j}(\Omega)\right)$, the corresponding trajectory $\gamma_{z}$ is defined for all $t \in(-\infty, 0]$ and the $\alpha$-limit $\alpha\left(\gamma_{z}\right)=\{b\}$.

(2) If there is $z \in \mathbb{D}$ such that the corresponding trajectory $\gamma_{z}$ is defined for all $t \in(-\infty, 0]$ and $\alpha\left(\gamma_{z}\right)=\{b\}$, then there exists a component $V_{j}(\Omega)$ of the invariant set of $\Omega$ which is a half-plane and $h(z) \in \overline{V_{j}(\Omega)}$.

There can be one or two half-open components of $V(\Omega)$ related to $b$. In either case, we have $v(\Omega)=\infty$ and, therefore, by Corollary $2.2, \varphi_{t}^{\prime}(b)=1$, for all $t>0$.

Those components of the invariant set which are strips are closely related to the collection of the repulsive boundary fixed points of $\Phi=\left(\varphi_{t}\right)$, that is, those points $a \in \partial \mathbb{D}$ such that $\varphi_{t}^{\prime}(a) \in(1,+\infty)$, for every $t>0$.

Theorem 2.5. Let $\Phi=\left(\varphi_{t}\right)$ be a semigroup of analytic functions with boundary $D W$-point $b$ and planar domain $\Omega=h(\mathbb{D})$.

(1) Let the component $V_{j}(\Omega)$ of $V(\Omega)$ be an open strip. There is a unique repulsive boundary fixed point $\xi\left(V_{j}(\Omega)\right)$ of $\Phi$ such that, for every $z \in h^{-1}\left(V_{j}(\Omega)\right)$, the corresponding trajectory $\gamma_{z}$ is defined for all $t \in(-\infty, 0]$ and $\alpha\left(\gamma_{z}\right)=$ $\left\{\xi\left(V_{j}(\Omega)\right)\right\}$.

(2) The map $\xi$ thus defined between open strip components of the invariant set and repulsive boundary fixed points is bijective.

(3) If there is $z \in \mathbb{D}$ such that the corresponding trajectory $\gamma_{z}$ is defined for all $t \in(-\infty, 0]$ and $\alpha\left(\gamma_{z}\right)$ is a repulsive boundary fixed point $\xi\left(V_{j}(\Omega)\right)$, then $h(z) \in \overline{V_{j}(\Omega)}$.

Now, we study the multipliers of the functions $\varphi_{t}$ at boundary fixed points $\xi$ which are $\alpha$-limits of trajectories of the system $\dot{w}=G(w)$. Clearly, we have three possibilities: $\xi$ is the DW-point, $\xi$ is repulsive or $\xi$ is superrepulsive. The repulsive case will be analyzed again by means of the strip components of the invariant set. 
Theorem 2.6. Let $\Phi=\left(\varphi_{t}\right)$ be a semigroup of analytic functions with planar domain $\Omega$. If $\xi \in \partial \mathbb{D}$ is a repulsive boundary fixed point of $\Phi$, then

$$
\varphi_{t}^{\prime}(\xi)=\exp \left(\frac{\pi}{\beta_{j}(\Omega)} t\right), \quad \text { for all } t \geq 0,
$$

where $\beta_{j}(\Omega)$ is the width (necessarily finite) of the strip $V_{j}(\Omega)$ of the invariant set of $\Omega$ associated to $\xi$.

These results can be translated to the context of the angular derivative of an analytic function. Cowen and Pommerenke [1982, Theorem 6.1] showed that if $\varphi$ is an analytic and univalent function in $\mathbb{D}$ with $\varphi(\mathbb{D}) \subset \mathbb{D}$, Denjoy-Wolff point $b \in \partial \mathbb{D}, \varphi^{\prime}(b)<1$ and $\xi_{1}, \xi_{2}, \ldots, \xi_{n}$ are distinct fixed points of $\varphi$ (different from $b)$, then

$$
\sum_{j=1}^{n} \frac{1}{\log \varphi^{\prime}\left(\xi_{j}\right)} \leq-\frac{1}{\log \varphi^{\prime}(b)}
$$

and, if equality holds, $\varphi$ can be embedded in a semigroup of analytic functions and $\mathbb{D} \backslash \varphi(\mathbb{D})$ consists of $n-1$ analytic arcs. Indeed, some geometric motivation of this inequality can be read in that paper.

Now, if we suppose that $\varphi$ can be embedded in a semigroup of analytic functions, by Theorems 2.1 and 2.6, we can guarantee that, for some $t>0$,

$$
\varphi^{\prime}(b)=\exp \left(-\frac{\pi}{v(\Omega)} t\right) \quad \text { and } \quad \varphi^{\prime}\left(\xi_{j}\right)=\exp \left(\frac{\pi}{\beta_{j}(\Omega)} t\right) .
$$

Therefore, we can always rewrite the inequality above as

$$
\sum_{j=1}^{n} \beta_{j}(\Omega) \leq v(\Omega) .
$$

This inequality reflects the geometric fact that the narrowest strip including $\Omega$ must contain the family of the disjoint strips $V_{j}(\Omega)$.

Finally, we treat the superrepulsive case.

Theorem 2.7. Let $\Phi=\left(\varphi_{t}\right)$ be a semigroup of analytic functions with boundary $D W$-point $b$ and planar domain $\Omega=h(\mathbb{D})$ as in the introduction. If there is a point $z \in \mathbb{D}$ such that

$$
h(z) \in\left(\bigcap_{t \geq 0}(\Omega+t)\right) \backslash\left(\bigcup_{j \in J} \overline{V_{j}(\Omega)}\right)
$$

and the corresponding trajectory $\gamma_{z}$ is defined for all $t \in(-\infty, 0]$ and $\alpha\left(\gamma_{z}\right)=\{\xi\}$, then $\xi$ is a superrepulsive boundary fixed point of $\Phi$ and $\gamma_{z}$ is the unique trajectory of the planar dynamical system with $\alpha\left(\gamma_{z}\right)=\{\xi\}$. 
This does not necessarily account for all superrepulsive fixed points: there are semigroups with a superrepulsive point $\xi$ where there is no trajectory $\gamma_{z}$ with $\alpha\left(\gamma_{z}\right)=\{\xi\}$. One example is the following: Consider the planar subset

$$
\Omega:=\left\{z \in \mathbb{C}:-\frac{1}{2}<\operatorname{Im} z<\exp (\operatorname{Re} z)-\frac{1}{2}\right\},
$$

and take a Riemann map $h$ from $\mathbb{D}$ onto $\Omega$ with $h(0)=0$. Now, the semigroup we are looking for is

$$
\varphi_{t}(z):=h^{-1}(h(z)+t), \quad z \in \mathbb{D} .
$$

The inequality of Cowen and Pommerenke mentioned above implies that the number of repulsive fixed points on the boundary of the unit disk is denumerable. However, the number of boundary fixed points can be uncountable (of course, most of them must be superrepulsive). To build such an example, consider the set of rational numbers of the interval $(-1,1)$, say $\{\alpha(n): n \in \mathbb{N}\}$, and the planar subset

$$
\Omega:=\{z \in \mathbb{C}:-1<\operatorname{Im} z<1\} \backslash\left(\bigcup_{n \in \mathbb{N}}((-\infty,-n]+\alpha(n) i)\right)
$$

and take a Riemann map $h$ from $\mathbb{D}$ onto $\Omega$ with $h(0)=0$. Now, by Theorem 2.7, the semigroup of analytic functions given by

$$
\varphi_{t}(z):=h^{-1}(h(z)+t), \quad z \in \mathbb{D}
$$

has a nondenumerable set of boundary fixed points. This example was suggested to the authors by Ricardo Pérez-Marco. We thank him for this example and other interesting remarks concerning this paper. Additional information about the amount of fixed points of analytic self-maps on $\mathbb{D}$ can be read in [Cowen and Pommerenke 1982, Section 2].

Our next step is the analysis of the slopes of the trajectories of the system. As before, this requires recalling some notation first and distinguishing between several cases. These cases are related to the evolution of the distance of a fixed point to the boundary of the domain $\Omega$ and it suggests to introduce the following notation:

$$
\delta_{\Omega}(w)=\inf \{|w-z|: z \in \partial \Omega\}, \quad w \in \Omega .
$$

Given a curve $\Gamma: t \in\left(s_{1}, s_{2}\right) \rightarrow \Gamma(t) \in \mathbb{D}$ with a singleton $\omega$-limit $\omega(\Gamma)=\{\zeta\} \subset$ $\partial \mathbb{D}$, the set of the slopes of $\Gamma$ when arriving at $\zeta$ will be denoted by $\operatorname{Slope}^{+}(\Gamma)$ and it is defined as the $\omega$-limit of the curve

$$
t \in\left(s_{1}, s_{2}\right) \rightarrow \operatorname{Arg}(1-\bar{\zeta} \Gamma(t)) \in\left(-\frac{\pi}{2}, \frac{\pi}{2}\right),
$$

where $\operatorname{Arg}(z)$ denotes the principal argument of $z$. It is well-known that $\operatorname{Slope}^{+}(\Gamma)$ is a nonempty, compact, and connected subset of $\left[-\frac{\pi}{2}, \frac{\pi}{2}\right]$ and we talk about the 
slope of $\Gamma$ at $\zeta$ if Slope ${ }^{+}(\Gamma)$ is a single point. Something similar can be done with $\alpha$-limits and the definition of Slope ${ }^{-}(\Gamma)$ is self-explanatory.

In view of the continuous version of the Denjoy-Wolff Theorem, we know that $\lim _{t \rightarrow+\infty} \gamma_{z}(t) \rightarrow b$, so we may ask about the slope of the forward trajectory $\gamma_{z}$ when arriving at $b$. Our next theorem answers precisely this question when $v(\Omega)$ is finite.

Theorem 2.8. Let $\Phi=\left(\varphi_{t}\right)$ be a semigroup of analytic functions with boundary $D W$-point $b$ and planar domain $\Omega$. If $v(\Omega)<\infty$, then, for each $z \in \mathbb{D}$, the set Slope $^{+}\left(\gamma_{z}\right)$ is a single point and it belongs to $\left(-\frac{\pi}{2}, \frac{\pi}{2}\right)$. That is, the (forward) trajectory $\gamma_{z}$ tends to $b$ with a fixed slope and never tangentially. Moreover, given $\alpha \in\left(-\frac{\pi}{2}, \frac{\pi}{2}\right)$, there is $z \in \mathbb{D}$ such that $\operatorname{Slope}^{+}\left(\gamma_{z}\right)=\{\alpha\}$.

The next theorem treats the case $v(\Omega)=+\infty$. We notice that, given $w \in \Omega$, the limit $\lim _{s \rightarrow+\infty} \delta_{\Omega}(w+s)$ always exists and belongs to $(0,+\infty]$.

Theorem 2.9. Let $\Phi=\left(\varphi_{t}\right)$ be a semigroup of analytic functions with boundary $D W$-point $b$, planar domain $\Omega$ and $\nu(\Omega)=+\infty$.

(1) If there is $w \in \Omega$ such that $\lim _{s \rightarrow+\infty} \delta_{\Omega}(w+s)=+\infty$, then all the sets Slope $^{+}\left(\gamma_{z}\right)$ are identical, when $z$ runs the whole $\mathbb{D}$.

(2) If there is $w \in \Omega$ such that $\lim _{s \rightarrow+\infty} \delta_{\Omega}(w+s) \in(0,+\infty)$, then all the trajectories $\gamma_{z}(z \in \mathbb{D})$ tends tangentially to $b$. That is, $\operatorname{Slope}^{+}\left(\gamma_{z}\right)$ is a single point and it is equal to $-\frac{\pi}{2}$ or $\frac{\pi}{2}$.

We conjecture that, in case (1) of this theorem, all the subsets Slope ${ }^{+}\left(\gamma_{z}\right)$ are indeed singletons, so it would be possible to speak about the common slope of the trajectories of the system.

We now study the slopes of backward trajectories. The analysis of the sets Slope $^{-}\left(\gamma_{z}\right)$ will be done first, when the $\alpha$-limit of $\gamma_{z}$ is the DW-point.

Theorem 2.10. Let $\Phi=\left(\varphi_{t}\right)$ be a semigroup of analytic functions with boundary $D W$-point $b$ and planar domain $\Omega$.

(1) If the $\alpha$-limit of a trajectory $\gamma_{z}$ is $b$, then the set $\operatorname{Slope}^{-}\left(\gamma_{z}\right)$ is a single point and it is equal to $-\frac{\pi}{2}$ or $\frac{\pi}{2}$. That is, the backward trajectory $\gamma_{z}$ tends tangentially to $b$.

(2) Given two trajectories $\gamma_{z_{1}}$ and $\gamma_{z_{2}}$ where $b$ is their $\alpha$-limit and such that $h\left(z_{1}\right)$ and $h\left(z_{2}\right)$ belong to the closure of the same half-plane component of $V(\Omega)$, then Slope $^{-}\left(\gamma_{z_{1}}\right)=\operatorname{Slope}^{-}\left(\gamma_{z_{2}}\right)$.

Finally, we study the slopes of the backward trajectories reaching a repulsive boundary fixed point.

Theorem 2.11. Let $\Phi=\left(\varphi_{t}\right)$ be a semigroup of analytic functions with boundary $D W$-point $b$ and planar domain $\Omega$ and $\xi\left(V_{j}(\Omega)\right)$ a repulsive boundary fixed point. 
(1) For every $z \in h^{-1}\left(V_{j}(\Omega)\right)$, the set Slope $^{-}\left(\gamma_{z}\right)$ is a single point and it belongs to $\left(-\frac{\pi}{2}, \frac{\pi}{2}\right)$. That is, the backward trajectory $\gamma_{z}$ tends to $\xi\left(V_{j}(\Omega)\right)$ with a fixed slope and never tangentially. Moreover, given $\alpha \in\left(-\frac{\pi}{2}, \frac{\pi}{2}\right)$, there is $z \in h^{-1}\left(V_{j}(\Omega)\right)$ such that Slope $^{-}\left(\gamma_{z}\right)=\{\alpha\}$.

(2) If there is $z \in \mathbb{D}$ such that the trajectory $\gamma_{z}$ is defined for all $t \in(-\infty, 0]$, $\alpha\left(\gamma_{z}\right)=\left\{\xi\left(V_{j}(\Omega)\right)\right\}$ and $h(z) \in \overline{V_{j}(\Omega)} \backslash V_{j}(\Omega)$, then Slope $^{-}\left(\gamma_{z}\right)$ is a single point and it is equal to $-\frac{\pi}{2}$ or $\frac{\pi}{2}$. That is, the backward trajectory $\gamma_{z}$ tends tangentially to $\xi\left(V_{j}(\Omega)\right)$.

\section{The hyperbolic metric}

We recall here some facts and notations about the hyperbolic metric; for detailed exposition and proofs, see [Shapiro 1993; Milnor 1999].

Definition 3.1. Given $p$ and $q$ two points of $\mathbb{D}$ and $\gamma:[a, b] \rightarrow \mathbb{D}$ a piecewise $C^{1}$ function with $\gamma(a)=p$ and $\gamma(b)=q$, we define the hyperbolic length of $\gamma$ as

$$
l_{\mathbb{D}}(\gamma)=2 \int_{a}^{b} \frac{\left|\gamma^{\prime}(t)\right| d t}{1-|\gamma(t)|^{2}} .
$$

The hyperbolic distance or Poincaré distance from $p$ to $q$ is the length of the shortest curve from $p$ to $q$, that is,

$$
\rho_{\mathbb{D}}(p, q)=\inf _{\gamma} l_{\mathbb{D}}(\gamma)
$$

where $\gamma$ runs through all piecewise $C^{1}$ curves from $p$ to $q$.

It is not difficult to see that the hyperbolic distance is an unbounded complete metric on $\mathbb{D}$ and that it induces its usual Euclidean topology. Moreover, there is a curve in $\mathbb{D}$ where the infimum that appears in the definition is attained.

Proposition 3.2. Let $p$ and $q$ be two points of the unit disk $\mathbb{D}$. Then the following assertions are true.

(1) The hyperbolic distance can be calculated using the pseudo-hyperbolic distance, namely

$$
\rho_{\mathbb{D}}(p, q)=\log \frac{1+d(p, q)}{1-d(p, q)}
$$

where $d(p, q)$ is the pseudo-hyperbolic distance between $p$ and $q$ given by

$$
d(p, q)=\left|\frac{p-q}{1-\bar{p} q}\right| .
$$

(2) If $\varphi$ is an automorphism of the unit disc, then

$$
\rho_{\mathbb{D}}(\varphi(p), \varphi(q))=\rho_{\mathbb{D}}(p, q) .
$$


(3) Every holomorphic self-map $\varphi$ of $\mathbb{D}$ is a contraction in the hyperbolic metric, that is,

$$
\rho_{\mathbb{D}}(\varphi(p), \varphi(q)) \leq \rho_{\mathbb{D}}(p, q) .
$$

The key points in the study of angular derivative using the hyperbolic distance are the following two results. The first one is a slight generalization of [Shapiro 1993, p. 159, equality (8)].

Proposition 3.3. Let $\varphi$ be a holomorphic self-map of $\mathbb{D}$. If $\xi \in \partial \mathbb{D}$ is a fixed point of $\varphi$ with $\varphi^{\prime}(\xi) \neq \infty$ and $\left(z_{n}\right)$ is a sequence in $\mathbb{D}$ such that $\lim _{n} \operatorname{Arg}\left(1-\bar{\xi} z_{n}\right)=$ $\theta \in\left(-\frac{\pi}{2}, \frac{\pi}{2}\right)$, then

$$
\lim _{n \rightarrow \infty} \rho_{\mathbb{D}}\left(z_{n}, \varphi\left(z_{n}\right)\right)=\log \frac{\left|\varphi^{\prime}(\xi)+e^{-2 i \theta}\right|+\left|\varphi^{\prime}(\xi)-1\right|}{\left|\varphi^{\prime}(\xi)+e^{-2 i \theta}\right|-\left|\varphi^{\prime}(\xi)-1\right|} .
$$

In particular, $\lim _{n \rightarrow \infty} \rho_{\mathbb{D}}\left(z_{n}, \varphi\left(z_{n}\right)\right) \geq\left|\log \left(\varphi^{\prime}(\xi)\right)\right|$ and if $\lim _{n} \operatorname{Arg}\left(1-\bar{\xi} z_{n}\right)=0$, then

$$
\lim _{n \rightarrow \infty} \rho_{\mathbb{D}}\left(z_{n}, \varphi\left(z_{n}\right)\right)=\left|\log \varphi^{\prime}(\xi)\right| .
$$

Proposition 3.4. Let $\varphi$ be a holomorphic self-map of $\mathbb{D}$. If $b \in \partial \mathbb{D}$ is the DenjoyWolff point of $\varphi$, and $z, z_{0}$ two points of $\mathbb{D}$ such that the sequence $\left(z_{n}\right)$, given by $z_{n+1}=\varphi\left(z_{n}\right)$ for all $n$, converges to $b$ nontangentially, then

$$
\lim _{n \rightarrow \infty} \frac{\rho_{\mathbb{D}}\left(z, z_{n}\right)}{n}=\left|\log \varphi^{\prime}(b)\right| .
$$

Proof. We know that

$$
\lim _{n \rightarrow \infty} \varphi\left(z_{n}\right)=b \quad \text { and } \quad \lim _{n \rightarrow \infty} \frac{\varphi\left(z_{n}\right)-b}{z_{n}-b}=\varphi^{\prime}(b) \in(0,1] .
$$

So,

$$
\lim _{n \rightarrow \infty} \frac{\left|z_{n+1}-b\right|}{\left|z_{n}-b\right|}=\lim _{n \rightarrow \infty}\left|\frac{\varphi\left(z_{n}\right)-b}{z_{n}-b}\right|=\varphi^{\prime}(b) .
$$

Therefore,

$$
\lim _{n \rightarrow \infty}\left|z_{n}-b\right|^{1 / n}=\varphi^{\prime}(b) .
$$

Since $\left(z_{n}\right)$ converges nontangentially to $b$, there is $k>1$ such that $\left|z_{n}-b\right| \leq$ $k\left(1-\left|z_{n}\right|\right)$ for all $n \in \mathbb{N}$. Hence

$$
\left|z_{n}-b\right|^{1 / n} \leq k^{1 / n}\left(1-\left|z_{n}\right|\right)^{1 / n} \leq k^{1 / n}\left(\left|z_{n}-b\right|\right)^{1 / n}
$$

for all $n$. Now, we have $\lim _{n \rightarrow \infty}\left(1-\left|z_{n}\right|\right)^{1 / n}=\varphi^{\prime}(b)$. Moreover

$$
\lim _{n \rightarrow \infty}\left(\frac{1+\left|z_{n}\right|}{1-\left|z_{n}\right|}\right)^{1 / n}=\frac{1}{\varphi^{\prime}(b)} \in[1, \infty)
$$


and we obtain

$$
\begin{aligned}
\lim _{n \rightarrow \infty} \frac{\rho_{\mathbb{D}}\left(0, z_{n}\right)}{n} & =\lim _{n \rightarrow \infty} \frac{\rho_{\mathbb{D}}\left(0,\left|z_{n}\right|\right)}{n}=\lim _{n \rightarrow \infty} \frac{1}{n} \log \frac{1+\left|z_{n}\right|}{1-\left|z_{n}\right|} \\
& =\log \frac{1}{\varphi^{\prime}(b)}=\left|\log \varphi^{\prime}(b)\right| .
\end{aligned}
$$

Finally, bearing in mind that

$$
\frac{\rho_{\mathbb{D}}\left(0, z_{n}\right)-\rho_{\mathbb{D}}(0, z)}{n} \leq \frac{\rho_{\mathbb{D}}\left(z, z_{n}\right)}{n} \leq \frac{\rho_{\mathbb{D}}(0, z)+\rho_{\mathbb{D}}\left(0, z_{n}\right)}{n},
$$

we obtain

$$
\lim _{n \rightarrow \infty} \frac{\rho_{\mathbb{D}}\left(z, z_{n}\right)}{n}=\lim _{n \rightarrow \infty} \frac{\rho_{\mathbb{D}}\left(0, z_{n}\right)}{n}=\left|\log \varphi^{\prime}(b)\right| .
$$

Remark. A result similar to the proposition can be given for backward iteration sequences for $\varphi$ that converge to a fixed point.

Let $h$ be an univalent function of the unit disk $\mathbb{D}$ onto a simply connected domain $\Omega \varsubsetneqq \mathbb{C}$. We shall use the function $h$ to transfer the notion of hyperbolic distance from $\mathbb{D}$ to $\Omega$. More precisely, we define the hyperbolic distance on $\Omega$ by

$$
\rho_{\Omega}(h(p), h(q)):=\rho_{\mathbb{D}}(p, q)
$$

for all $p, q \in \mathbb{D}$. Moreover, given $\Gamma$ a piecewise $C^{1}$ curve in $\Omega$, the hyperbolic length of $\Gamma$ is given by $l_{\Omega}(\Gamma):=l_{\mathbb{D}}\left(h^{-1}(\Gamma)\right)$. Thus, the hyperbolic metric in $\Omega$ is invariant under the action of conformal automorphisms of $\Omega$ and induces the usual Euclidean topology.

A first approach to the relation between the geometry of the domain $\Omega$ and the hyperbolic distance involves the inequalities

$$
\frac{1}{2} \int_{\Gamma} \frac{|d w|}{\delta_{\Omega}(w)} \leq l_{\Omega}(\Gamma) \leq 2 \int_{\Gamma} \frac{|d w|}{\delta_{\Omega}(w)} .
$$

The first inequality leads to an estimate of how the hyperbolic distance increase as one moves toward the boundary.

Proposition 3.5 (Distance Lemma [Shapiro 1993, p. 157]). If $\Omega \varsubsetneqq \mathbb{C}$ is a simply connected domain, then for $P, Q \in \Omega$, we have

$$
\rho_{\Omega}(P, Q) \geq \frac{1}{2} \log \left(1+\frac{|P-Q|}{\min \left\{\delta_{\Omega}(P), \delta_{\Omega}(Q)\right\}}\right) .
$$

If we have two simply connected domains $\Omega_{1}$ and $\Omega_{2}$ such that $\Omega_{1} \subset \Omega_{2} \subsetneq \mathbb{C}$, we have a relation between their corresponding hyperbolic metrics. Take $h_{1}$ a Riemann 
map of $\Omega_{1}$ and $h_{2}$ a Riemann map of $\Omega_{2}$. Then $\varphi=h_{2}^{-1} \circ h_{1}$ is an analytic self-map of $\mathbb{D}$. So, it is a contraction in the hyperbolic metric, that is,

$$
\rho_{\mathbb{D}}(\varphi(p), \varphi(q)) \leq \rho_{\mathbb{D}}(p, q)
$$

for all $p, q \in \mathbb{D}$. In particular, given $P, Q \in \Omega_{1}$, we have

$$
\begin{aligned}
\rho_{\Omega_{2}}(P, Q) & =\rho_{\mathbb{D}}\left(h_{2}^{-1}(P), h_{2}^{-1}(Q)\right)=\rho_{\mathbb{D}}\left(\varphi \circ h_{1}^{-1}(P), \varphi \circ h_{1}^{-1}(Q)\right) \\
& \leq \rho_{\mathbb{D}}\left(h_{1}^{-1}(P), h_{1}^{-1}(Q)\right)=\rho_{\Omega_{1}}(P, Q) .
\end{aligned}
$$

So we have the following well-known result.

Proposition 3.6. Given two simply connected domains $\Omega_{1}$ and $\Omega_{2}$ such that $\Omega_{1} \subset$ $\Omega_{2} \subsetneq \mathbb{C}$ and $P, Q \in \Omega_{1}$, we have

$$
\rho_{\Omega_{2}}(P, Q) \leq \rho_{\Omega_{1}}(P, Q) .
$$

\section{Fixed points and nontangential convergence}

In this section we present a first approach to the relationship between fixed points of the semigroup $\left(\varphi_{t}\right)$ and the geometry of the domain $\Omega$. Roughly speaking, each horizontal half-line in $\Omega$ induces a curve in the unit disc whose end point is a fixed point of the functions of the semigroup, and the distance from the points of the half-line to the boundary of $\Omega$ determines whether or not convergence is tangential.

The starting point is the following well-known result: if $\Gamma:[0, \infty) \rightarrow \Omega$ is any curve with $\lim _{s \rightarrow \infty} \Gamma(s)=\infty$ (and $h$ is an univalent function on $\mathbb{D}$ such that $\Omega=h(\mathbb{D}))$, then there exists $w \in \partial \mathbb{D}$ such that $\lim _{s \rightarrow \infty} h^{-1}(\Gamma(s))=w$ [Shapiro 1993, p. 162]. Our first result is a necessary condition to guarantee that, given two different curves in $\Omega$, the corresponding curves in the unit disk have the same end point.

Lemma 4.1. Suppose $h$ is a univalent function on $\mathbb{D}$ and $\Omega=h(\mathbb{D})$ and that $\Gamma_{i}:[0, \infty) \rightarrow \Omega, i=1,2$, are two Jordan arcs with $\Gamma_{1}(s) \neq \Gamma_{2}\left(s^{\prime}\right)$ for all $\left(s, s^{\prime}\right) \neq$ $(0,0), \Gamma_{1}(0)=\Gamma_{2}(0), \lim _{s \rightarrow \infty} \Gamma_{1}(s)=\lim _{s \rightarrow \infty} \Gamma_{2}(s)=\infty$, and that one of the two connected components of the complement of $\Gamma_{1}[0, \infty) \cup \Gamma_{2}[0, \infty)$ in $\mathbb{C}$ is included in $\Omega$. Then $\lim _{s \rightarrow \infty} h^{-1}\left(\Gamma_{1}(s)\right)=\lim _{s \rightarrow \infty} h^{-1}\left(\Gamma_{2}(s)\right)$.

Proof. Denote by $\Theta$ the connected component of the complement of $\Gamma_{1}[0, \infty) \cup$ $\Gamma_{2}[0, \infty)$ that is included in $\Omega$ and

$$
\omega(\Theta):=\left\{b \in \overline{\mathbb{D}}: \text { there is }\left\{w_{n}\right\} \subset \bar{\Theta}, \text { with }\left|w_{n}\right| \nearrow \infty \text { and } h^{-1}\left(w_{n}\right) \rightarrow b\right\} .
$$

Notice that $\partial \Theta$ is a Jordan curve in the Riemann sphere whose boundary is

$$
\Gamma_{1}[0, \infty) \cup \Gamma_{2}[0, \infty) \cup\{\infty\} .
$$


Since $h$ is continuous on $\mathbb{D}$, we have $\omega(\Theta) \subseteq \partial \mathbb{D}$. Moreover, $\lim _{s \rightarrow \infty} h^{-1}\left(\Gamma_{1}(s)\right)$ and $\lim _{s \rightarrow \infty} h^{-1}\left(\Gamma_{2}(s)\right)$ are in $\omega(\Theta)$. So the proof is finished if we obtain that $\omega(\Theta)$ is a single point. First of all, notice that $\omega(\Theta)$ is closed.

Now we focus our attention on checking that $\omega(\Theta)$ is connected. Suppose that there are two closed, disjoint, and nonempty subsets $A$ and $B$ of $\omega(\Theta)$ such that $\omega(\Theta)=A \cup B$. Let $K=d(A, B)>0$. For each natural number $n$, we can take $a_{n} \in A, b_{n} \in B$, and $w_{n}, \tilde{w}_{n} \in \bar{\Theta}$, such that

$$
\left|a_{n}-h^{-1}\left(w_{n}\right)\right|<K / 3, \quad\left|b_{n}-h^{-1}\left(\tilde{w}_{n}\right)\right|<K / 3,
$$

and $\left|\tilde{w}_{n}\right|>\left|w_{n}\right|>\max \left\{\left|\tilde{w}_{n-1}\right|, n\right\}$. In particular,

$$
d\left(h^{-1}\left(w_{n}\right), A\right)<K / 3 \quad \text { and } \quad d\left(h^{-1}\left(\tilde{w}_{n}\right), A\right)>2 K / 3 \quad \text { for all } n .
$$

Claim. Given $w_{n}$ and $\tilde{w}_{n}$ in $\bar{\Theta}$ with $\left|w_{n}\right| \nearrow \infty$ and $\left|\tilde{w}_{n}\right| \nearrow \infty$, there is a curve $\gamma_{n}$ in $\bar{\Theta}$, for each $n$, from $w_{n}$ to $\tilde{w}_{n}$ such that $\min _{w \in \gamma_{n}}|w|$ goes to $\infty$ as $n$ goes to $\infty$. (The claim will be proved after the proof of the lemma is complete.)

By the continuity of the function $h$ and taking $\gamma_{n}$ the curve given by the claim, there is $z_{n} \in \gamma_{n}$ such that $d\left(h^{-1}\left(z_{n}\right), A\right)=K / 2$ and $\left|z_{n}\right| \rightarrow \infty$. This is a contradiction since any point of accumulation of the sequence $\left(h^{-1}\left(z_{n}\right)\right)$ is in $\omega(\Theta)$ but it is neither in $A$ nor in $B$.

Summing up, $\omega(\Theta)$ is nonempty, connected, and compact. Suppose that $\omega(\Theta)$ is not a single point. On the one hand, recall that $h$ has radial limits a.e. on $\partial \mathbb{D}$ [Shapiro 1993, p. 162]. On the other hand, using Lehto-Virtanen Theorem we obtain that $\omega(\Theta) \varsubsetneqq \partial \mathbb{D}$. So, take a nontrivial subarc $\Upsilon$ of $\omega(\Theta)$ such that if $b \in \Upsilon$, then $-b \notin \omega(\Theta)$. Take $w_{n}$ and $\tilde{w}_{n}$ in $\bar{\Theta}$ with $\left|w_{n}\right| \nearrow \infty$ and $\left|\tilde{w}_{n}\right| \nearrow \infty$ such that $h^{-1}\left(w_{n}\right) \rightarrow b_{1}$ and $h^{-1}\left(\tilde{w}_{n}\right) \rightarrow b_{2}$, where $b_{1}$ and $b_{2}$ are the extreme points of $\omega(\Theta)$. By the claim, for each $n$, there is a curve $\gamma_{n}$ in $\bar{\Theta}$, from $w_{n}$ to $\tilde{w}_{n}$, such that $\min _{w \in \gamma_{n}}|w|$ goes to $\infty$ as $n$ goes to $\infty$. So, if $b \in \Upsilon$ is different from the extreme points of $\omega(\Theta)$ and $h$ has radial limit in $b$, there is $r_{n} \rightarrow 1$ such that $h\left(r_{n} b\right) \in \gamma_{n}$. Hence $h\left(r_{n} b\right) \rightarrow \infty$. Therefore, we have that $\lim _{r \rightarrow 1} h(r b)=\infty$. That is, $h$ has a radial limit equal to $\infty$ a.e. on $\Upsilon$. But this contradicts that this radial limit is not a.e. constant on any subarc of $\partial \mathbb{D}$ [Shapiro 1993, p. 162].

Proof of the claim. Since $\Theta$ is a Jordan domain in the Riemann sphere, its Riemann mapping $\mu$ has a bijective and bicontinuous extension to the closed unit disc [Milnor 1999, Theorem 17.16]. Denote by $\tilde{\gamma}_{n}$ the segment $\left[\mu^{-1}\left(w_{n}\right), \mu^{-1}\left(\tilde{w}_{n}\right)\right] \subset \overline{\mathbb{D}}$. The curve we are looking for is $\gamma_{n}=\mu\left(\tilde{\gamma}_{n}\right)$. In effect, take $z_{n} \in \gamma_{n}$ such that $\left|z_{n}\right|=\min _{w \in \gamma_{n}}|w|$. Then there is $\lambda_{n} \in[0,1]$ such that $\mu^{-1}\left(z_{n}\right)=\lambda_{n} \mu^{-1}\left(w_{n}\right)+$ $\left(1-\lambda_{n}\right) \mu^{-1}\left(\tilde{w}_{n}\right)$. Since $\left(w_{n}\right)$ and $\left(\tilde{w}_{n}\right)$ converge to $\infty$, we see that $\left(\mu^{-1}\left(z_{n}\right)\right)$ converges to $\mu^{-1}(\infty)$. Therefore, $\left(z_{n}\right)$ goes to $\infty$ as $n$ tends to $\infty$. 
Now, it is time to introduce the relationship between half-lines in $\Omega$ and fixed points of the functions $\varphi_{t}$.

Lemma 4.2. Fix $t>0$ and $a \in \Omega$. Take $\kappa \in\{-1,1\}$. Suppose that the curve $\Gamma(s)=a+\kappa s t$, with $s \geq 0$, is in $\Omega$. Then there is a point $\xi \in \partial \mathbb{D}$ such that $\lim _{s \rightarrow \infty} h^{-1}(a+\kappa s t)=\xi$ and $\xi$ is a fixed point of $\varphi_{t}$.

Proof. We know that there is a point $\xi \in \partial \mathbb{D}$ such that $\lim _{s \rightarrow \infty} h^{-1}(a+\kappa s t)=\xi$. Notice that

$$
\begin{aligned}
\lim _{s \rightarrow \infty} \varphi_{t}\left(h^{-1}(a+\kappa s t)\right) & =\lim _{s \rightarrow \infty} h^{-1}(a+\kappa s t+t)=\lim _{s \rightarrow \infty} h^{-1}(a+\kappa(s+\kappa) t) \\
& =\lim _{s \rightarrow \infty} h^{-1}(a+\kappa s t)=\xi .
\end{aligned}
$$

Therefore, by Lindelöf's Theorem, $\xi$ is a fixed point of $\varphi_{t}$ and $\angle \lim _{z \rightarrow \xi} \varphi_{t}(z)=\xi$.

To calculate the derivative at a fixed point we use Propositions 3.3 and 3.4. We have to estimate $\rho_{\mathbb{D}}\left(z_{1}, \varphi_{t}\left(z_{2}\right)\right)$ as $\varphi_{t}\left(z_{2}\right)$ converges to $\xi$ nontangentially. If we take $z_{i}=h^{-1}\left(a+\kappa s_{i} t\right)$, we have

$$
\rho_{\mathbb{D}}\left(h^{-1}\left(a+\kappa s_{1} t\right), \varphi_{t}\left(h^{-1}\left(a+\kappa s_{2} t\right)\right)\right)=\rho_{\Omega}\left(a+\kappa s_{1} t, a+\kappa s_{2} t+t\right) .
$$

The way to calculate this hyperbolic distance depends on the type (repulsive or attractive) of fixed point we have. But first we have to check that $h^{-1}(a+\kappa s t)$ converges nontangentially to $\xi$. On the one hand, when $\kappa=1$ and the derivative $\varphi_{t}^{\prime}(\xi)$ is less than 1 , this will be done using the following result:

Lemma 4.3 [Cowen 1981, Lemma 2.1]. Suppose $\varphi$ be a holomorphic self-map of $\mathbb{D}$, and has Denjoy-Wolff point $b \in \partial \mathbb{D}$ with $\varphi^{\prime}(b)<1$. Then, for any $z$ in $\mathbb{D}$, the sequence $\left(\varphi_{n}(z)\right)$ converges nontangentially to $b$.

On the other hand, nontangential convergence will be characterized in terms of the euclidean distance from the point $a+\kappa s t$ to the boundary of $\Omega$. First we need the following lemma that, roughly speaking, says that to each fixed point with finite derivative corresponds a "tube" in $\Omega$.

Lemma 4.4. Let $\varphi$ be a holomorphic self-map of $\mathbb{D}$, with $\varphi(z)=h^{-1}(h(z)+\lambda), h$ univalent, $\lambda>0, \Omega=h(\mathbb{D})$, and $\xi \in \partial \mathbb{D}$ a fixed point of $\varphi$ with $\varphi^{\prime}(\xi) \neq \infty$. Then there is a positive number $\varepsilon=\varepsilon(\xi)$ such that $\delta_{\Omega}(h(r \xi)) \geq \varepsilon$ for all $r \in[0,1)$.

Proof. To simplify the notation, we set

$$
d=\varphi^{\prime}(\xi)=\lim _{r \rightarrow 1^{-}} \frac{\xi-\varphi(r \xi)}{\xi-r \xi} \in(0, \infty)
$$


Thus there is $r_{0}<1$, such that $r_{0}<r<1$ implies $|\xi-\varphi(r \xi)| \leq(d+1)(1-r)$. So, we have

$$
|r \xi-\varphi(r \xi)| \leq|\xi-\varphi(r \xi)|+|\xi-r \xi| \leq(d+2)(1-r),
$$

whenever $r>r_{0}$. Hence, estimating the hyperbolic distance by integrating along the segment $[r \xi, \varphi(r \xi)]$, we get

$$
\rho_{\mathbb{D}}(r \xi, \varphi(r \xi)) \leq 2 \int_{[r \xi, \varphi(r \xi)]} \frac{d s(x)}{\delta_{\mathbb{D}}(x)} .
$$

We have to distinguish two cases. On the one hand, if $|\varphi(r \xi)| \leq r$, we have

$$
\rho_{\mathbb{D}}(r \xi, \varphi(r \xi)) \leq 2 \int_{[r \xi, \varphi(r \xi)]} \frac{d s(x)}{\delta_{\mathbb{D}}(x)} \leq 2 \frac{|r \xi-\varphi(r \xi)|}{1-r} \leq 2(d+2) .
$$

On the other hand, if $|\varphi(r \xi)|>r$, we have

$$
\rho_{\mathbb{D}}(r \xi, \varphi(r \xi)) \leq 2 \int_{[r \xi, \varphi(r \xi)]} \frac{d s(x)}{\delta_{\mathbb{D}}(x)} \leq 2 \frac{|r \xi-\varphi(r \xi)|}{1-|\varphi(r \xi)|} \leq 2(d+2) \frac{1-r}{1-|\varphi(r \xi)|},
$$

where the second inequality follows from Julia's Lemma [Cowen and MacCluer 1995, p. 49]; from this we then get

$$
\rho_{\mathbb{D}}(r \xi, \varphi(r \xi)) \leq 2(d+2) d \frac{1+|\varphi(r \xi)|}{1+r} \frac{(1-r)^{2}}{|\xi-\varphi(r \xi)|^{2}} \leq 4(d+2) d\left|\frac{\xi-r \xi}{\xi-\varphi(r \xi)}\right|^{2} .
$$

Since

$$
\lim _{r \rightarrow 1^{-}} \frac{\xi-r \xi}{\xi-\varphi(r \xi)}=\frac{1}{d}
$$

we see that

$$
\lim _{r \rightarrow 1} 4(d+2) d\left|\frac{\xi-r \xi}{\xi-\varphi(r \xi)}\right|^{2}=4 \frac{d+2}{d} .
$$

Hence, there is $r_{0} \leq r_{1}<1$ such that $\rho_{\mathbb{D}}(r \xi, \varphi(r \xi))$ is bounded for $r \in\left(r_{1}, 1\right)$.

So far, we have found that there is a constant $M>0$ such that

$$
\rho_{\Omega}(h(r \xi), h(r \xi)+\lambda)=\rho_{\mathbb{D}}(r \xi, \varphi(r \xi)) \leq M \quad \text { for all } r \in[0,1) .
$$

Now, by the Distance Lemma (Proposition 3.5),

$$
2 M \geq \log \left(1+\frac{|h(r \xi)-h(r \xi)-\lambda|}{\min \left\{\delta_{\Omega}(h(r \xi)), \delta_{\Omega}(h(r \xi)+\lambda)\right\}}\right) \geq \log \left(1+\frac{\lambda}{\delta_{\Omega}(h(r \xi))}\right) .
$$

That is, $\delta_{\Omega}(h(r \xi)) \geq \lambda /\left(e^{2 M}-1\right)>0$ for all $r$.

It is worth mentioning that this lemma implies that if $\xi \in \partial \mathbb{D}$ is a fixed point of $\varphi$ with $\varphi^{\prime}(\xi) \neq \infty$, then $\lim _{r \rightarrow 1^{-}} h(r \xi)=\infty$. 
Now we can give a sufficient condition to get the nontangential convergence. Since $\varphi_{t}(r \xi)$ converges nontangentially (as $r \rightarrow 1$ ) to the fixed point $\xi$, we will compare the curve $r \mapsto \varphi_{t}(r \xi)$ with the curve $s \mapsto h^{-1}(a+\kappa s t)$.

Proposition 4.5. Let $\left(\varphi_{t}\right)$ be a semigroup of analytic functions with planar domain $\Omega=h(\mathbb{D})$. Let $\xi \in \partial \mathbb{D}$ be a nonsuperrepulsive $\left(\varphi_{t}^{\prime}(\xi) \neq \infty\right.$ for all $\left.t>0\right)$ fixed point of $\left(\varphi_{t}\right)$. Take $\kappa \in\{-1,1\}$. Suppose that there is $s_{0}$ such that

(1) there is $\alpha>0$ such that $\delta_{\Omega}(a+\kappa s t) \geq \alpha$ for all $s \geq s_{0}$;

(2) there is $\beta<\infty$ such that for each $s \geq s_{0}$, there is $r(s)$ such that $\operatorname{Re} h(r(s) \xi)=$ $a+\kappa s t,[a+\kappa t s, h(r(s) \xi)+t] \subset \Omega$, and $|h(r(s) \xi)-(a+\kappa s t)| \leq \beta$.

Then $h^{-1}(a+\kappa s t)$ converges nontangentially to $\xi$ as $s$ goes to $\infty$.

Proof. Bearing in mind [Shapiro 1993, p. 171, Exercise 4], it is enough to find a constant $M$ such that $\rho_{\mathbb{D}}\left(h^{-1}(a+\kappa t s), \gamma\right) \leq M$ for all $s$ large enough where $\gamma$ is the segment $(-\xi, \xi)$. Fix $s>0$. We have

$$
\begin{aligned}
\rho_{\mathbb{D}}\left(h^{-1}(a+\kappa t s), \gamma\right) & \leq \inf _{0 \leq r<1} \rho_{\mathbb{D}}\left(h^{-1}(a+\kappa t s), r \xi\right) \\
& \leq \inf _{0 \leq r<1}\left(\rho_{\mathbb{D}}\left(r \xi, \varphi_{t}(r \xi)\right)+\rho_{\mathbb{D}}\left(h^{-1}(a+\kappa t s), \varphi_{t}(r \xi)\right)\right) .
\end{aligned}
$$

Since $\varphi_{t}(r \xi)$ converges nontangentially (as $r \rightarrow 1$ ) to the fixed point $\xi$, there is a constant $m$ such that $\rho_{\mathbb{D}}\left(r \xi, \varphi_{t}(r \xi)\right) \leq m$. So we just have to control

$$
\inf _{0 \leq r<1} \rho_{\mathbb{D}}\left(h^{-1}(a+\kappa t s), \varphi_{t}(r \xi)\right)
$$

as $s$ tends to $\infty$. Notice that

$$
\begin{aligned}
\inf _{0 \leq r<1} \rho_{\mathbb{D}}\left(h^{-1}(a+\kappa t s), \varphi_{t}(r \xi)\right) & =\inf _{0 \leq r<1} \rho_{\Omega}(a+\kappa t s, h(r \xi)+t) \\
& \leq 2 \inf _{0 \leq r<1} \inf _{\Gamma} \int_{\Gamma} \frac{d s(x)}{\delta_{\Omega}(x)},
\end{aligned}
$$

where the last infimum is taken over all curves that goes from $a+\kappa t s$ to $h(r \xi)+t$ in $\Omega$. Take $r(s)$ such that $\operatorname{Re} h(r(s) \xi)+t=\kappa s t$. Now we estimate the hyperbolic distance by integrating along the segment $[a+\kappa t s, h(r(s) \xi)+t]$ :

$$
\begin{aligned}
& \inf _{0 \leq r<1} \rho_{\mathbb{D}}\left(h^{-1}(a+\kappa t s), \varphi_{t}(r \xi)\right) \\
& \quad \leq 2 \inf _{0 \leq r<1} \inf _{\Gamma} \int_{\Gamma} \frac{d s(x)}{\delta_{\Omega}(x)} \leq 2 \int_{[a+\kappa t s, h(r(s) \xi)+t]} \frac{d s(x)}{\delta_{\Omega}(x)} \\
& \quad \leq 2 \frac{|a+\kappa t s-h(r(s) \xi)-t|}{\min \left\{\delta_{\Omega}(a+\kappa t s), \delta_{\Omega}(h(r(s) \xi)+t)\right\}} \leq 2 \frac{|a+\kappa t s-h(r(s) \xi)-t|}{\min \left\{\delta_{\Omega}(a+\kappa t s), \delta_{\Omega}(h(r(s) \xi))\right\}} .
\end{aligned}
$$


Now, by Lemma 4.4, there is $\varepsilon=\varepsilon(\xi)$ such that $\delta_{\Omega}(h(r \xi)) \geq \varepsilon$ for all $r \in[0,1)$. Therefore

$$
\rho_{\mathbb{D}}\left(h^{-1}(a+\kappa t s), \gamma\right) \leq 2 \frac{\beta+t}{\min \{\alpha, \varepsilon\}}
$$

for $s \geq s_{0}$. Therefore, $\rho_{\mathbb{D}}\left(h^{-1}(a+\kappa t s), \gamma\right)$ is bounded as $s$ goes to $\infty$.

Lemma 4.6. Let $\varphi$ be a holomorphic self-map of $\mathbb{D}$, with $\varphi(z)=h^{-1}(h(z)+\lambda), h$ univalent, $\lambda>0$ and $\Omega=h(\mathbb{D})$ with Denjoy-Wolff point equal to $b \in \partial \mathbb{D}$ and such that $\varphi^{\prime}(b)=1$. If $\left(p_{n}\right)$ is a sequence in $\mathbb{D}$ which converges nontangentially to $b$, then

$$
\lim _{n \rightarrow \infty} \delta_{\Omega}\left(h\left(p_{n}\right)\right)=\infty .
$$

Proof. Take a subsequence $\left(p_{n_{k}}\right)$ of $\left(p_{n}\right)$ such that $\lim _{k} \operatorname{Arg}\left(1-\bar{b} p_{n_{k}}\right)=\theta$. Then $\theta \in\left(-\frac{\pi}{2}, \frac{\pi}{2}\right)$ and, by Proposition 3.3,

$$
\begin{aligned}
\lim _{n \rightarrow \infty} \rho_{\Omega}\left(h\left(p_{n_{k}}\right), h\left(p_{n_{k}}\right)+\lambda\right) & =\lim _{n \rightarrow \infty} \rho_{\mathbb{D}}\left(p_{n_{k}}, \varphi\left(p_{n_{k}}\right)\right) \\
& =\log \frac{\left|\varphi^{\prime}(b)+e^{-2 i \theta}\right|+\left|\varphi^{\prime}(b)-1\right|}{\left|\varphi^{\prime}(b)+e^{-2 i \theta}\right|-\left|\varphi^{\prime}(b)-1\right|}=\log 1=0 .
\end{aligned}
$$

Because the choice of a subsequence was arbitrary we can deduce from this that $\lim _{n \rightarrow \infty} \rho_{\Omega}\left(h\left(p_{n}\right), h\left(p_{n}\right)+\lambda\right)=0$. Moreover, by the Distance Lemma (Proposition $3.5)$, we have

$$
\begin{aligned}
\rho_{\Omega}\left(h\left(p_{n}\right), h\left(p_{n}\right)+\lambda\right) & \geq \frac{1}{2} \log \left(1+\frac{\left|h\left(p_{n}\right)-h\left(p_{n}\right)-\lambda\right|}{\min \left\{\delta_{\Omega}\left(h\left(p_{n}\right)\right), \delta_{\Omega}\left(h\left(p_{n}\right)+\lambda\right)\right\}}\right) \\
& \geq \frac{1}{2} \log \left(1+\frac{\lambda}{\delta_{\Omega}\left(h\left(p_{n}\right)\right)}\right) .
\end{aligned}
$$

Hence, $\lim _{n \rightarrow \infty} \delta_{\Omega}\left(h\left(p_{n}\right)\right)=\infty$.

\section{Proofs of the main results}

Proof of Theorem 2.1. Siskakis [1985, Theorem 1.7] proved that there is a number $0<r \leq 1$ such that $\varphi_{t}^{\prime}(b)=r^{t}$ for all $t>0$. Therefore, it is clear that (2) is equivalent to (3).

$(1) \Rightarrow(2)$. By assumption, there is a real number $a$ such that

$$
\Omega \subseteq \Theta:=\left\{z \in \mathbb{C}: a-\frac{1}{2} v(\Omega)<\operatorname{Im} z<a+\frac{1}{2} v(\Omega)\right\} .
$$

A Riemann map of $\Theta$ is given by $\Phi(z)=(\nu(\Omega) / \pi) \log ((1-z) /(1+z))+a i$. Fix $t>0$. Take $c \in \mathbb{R}$ such that $a i+c \in \Omega$. Consider the points $w_{n}=a i+c+n t$. Notice that $w_{n} \in \Omega$. We will apply Proposition 3.4 with $z_{n}=h^{-1}\left(w_{n}\right)$ and $\varphi=\varphi_{t}$. So we have to check that $\left(z_{n}\right)$ converges nontangentially to $b$. To obtain this we will apply Proposition 4.5 with $\xi=1$ and $\kappa=1$. Recall that $0<\varphi_{t}^{\prime}(b) \leq 1$. On 
the one hand, in our case, the function $s \mapsto \delta_{\Omega}(a i+c+s t)$ is nondecreasing. So, there is $\alpha>0$ such that $\delta_{\Omega}(a i+c+s t) \geq \alpha$ for all $s \geq 0$. On the other hand, $h(r b)$ converges to $\infty$ and, bearing in mind that $b$ is attractive and that $\Omega \subseteq \Theta$, we see that $\operatorname{Re} h(r b)$ converges to $+\infty$ as $r$ tends to 1 . So, the assumption (2) of Proposition 4.5 is satisfied for $s$ large enough. Hence we can apply this proposition and obtain that $\left(z_{n}\right)$ converges nontangentially to $b$. Now we are ready to apply Proposition 3.4:

$$
\begin{aligned}
-\log \varphi_{t}^{\prime}(b) & =\lim _{n \rightarrow \infty} \frac{\rho_{\mathbb{D}}\left(h^{-1}(a i+c), \varphi_{t}\left(h^{-1}\left(w_{n}\right)\right)\right)}{n} \\
& =\lim _{n \rightarrow \infty} \frac{\rho_{\Omega}(a i+c, a i+c+n t+t)}{n} .
\end{aligned}
$$

By Proposition 3.6, we have

$$
\lim _{n \rightarrow \infty} \frac{\rho_{\Omega}(a i+c, a i+c+n t+t)}{n} \geq \lim _{n \rightarrow \infty} \frac{\rho_{\Theta}(a i+c, a i+c+n t+t)}{n} .
$$

Therefore

$$
\begin{aligned}
-\log \varphi_{t}^{\prime}(b) & \geq \lim _{n \rightarrow \infty} \frac{\rho_{\Theta}(a i+c, a i+c+n t+t)}{n} \\
& =\lim _{n \rightarrow \infty} \frac{\rho_{\mathbb{D}}\left(\Phi^{-1}(a i+c), \Phi^{-1}(a i+c+n t+t)\right)}{n} \\
& =\lim _{n \rightarrow \infty} \frac{1}{n} \rho_{\mathbb{D}}\left(\frac{1-\exp (c \pi / v(\Omega))}{1+\exp (c \pi / v(\Omega))}, \frac{1-\exp ((c+n t+t) \pi / v(\Omega))}{1+\exp ((c+n t+t) \pi / v(\Omega))}\right) \\
& =\lim _{n \rightarrow \infty} \frac{1}{n} \log \left(\frac{1+\left|\frac{1-\exp ((n t+t) \pi / v(\Omega))}{1+\exp ((n t+t) \pi / v(\Omega))}\right|}{1-\left|\frac{1-\exp ((n t+t) \pi / v(\Omega))}{1+\exp ((n t+t) \pi / v(\Omega))}\right|}\right) \\
& =\lim _{n \rightarrow \infty} \frac{1}{n} \log \exp \left(\frac{\pi t}{v(\Omega)}(n+1)\right)=\frac{\pi}{v(\Omega)} t .
\end{aligned}
$$

That is, $\varphi_{t}^{\prime}(b) \leq \exp (-\pi t / v(\Omega))<1$ for all $t>0$.

(2) $\Rightarrow$ (1) Fix $t>0$ and take an arbitrary $\lambda<v(\Omega)$. We will prove shortly that $\varphi_{t}^{\prime}(b) \geq \exp (-\pi t / \lambda)$, that is, $\lambda \leq \pi t /\left(-\log \varphi_{t}^{\prime}(b)\right)$. Therefore,

$$
\nu(\Omega) \leq \frac{\pi t}{-\log \varphi_{t}^{\prime}(b)}<\infty .
$$

Moreover, once we have obtained this inequality and bearing in mind the proof of the implication $(1) \Rightarrow(2)$, we will have shown that $v(\Omega)<\infty$ implies $\varphi_{t}^{\prime}(b)=$ $\exp (-\pi t / v(\Omega))$.

To obtain that $\varphi_{t}^{\prime}(b) \geq \exp (-\pi t / \lambda)$, we again apply Proposition 3.4. Take a point $a$ such that $\left[a-\frac{1}{2} \lambda i, a+\frac{1}{2} \lambda i\right] \subset \Omega$. Bearing in mind the geometric properties of 
$\Omega$, we have

$$
\Theta=\left\{z \in \mathbb{C}: \operatorname{Re} z>\operatorname{Re} a \text { and } \operatorname{Im} a-\frac{1}{2} \lambda<\operatorname{Im} z<\operatorname{Im} a+\frac{1}{2} \lambda\right\} \subseteq \Omega .
$$

Recall that a Riemann map of $\Theta$ is given by

$$
\Psi(z)=-i \frac{\lambda}{\pi} \sin ^{-1}\left(i \frac{1-z}{1+z}\right)+a .
$$

In fact,

$$
\Psi^{-1}(w)=\frac{1+i \sin (i \pi(w-a) / \lambda)}{1-i \sin (i \pi(w-a) / \lambda)} .
$$

Consider the points $w_{n}=a+n t \in \Theta \subset \Omega$. We will apply Proposition 3.4 with $z_{n}=h^{-1}\left(w_{n}\right)$ and $\varphi=\varphi_{t}$. First of all, since $\varphi_{t}^{\prime}(b)<1$, Lemma 4.3 says that $\left(z_{n}\right)$ converges nontangentially to $b$. Hence

$-\log \varphi_{t}^{\prime}(b)=\lim _{n \rightarrow \infty} \frac{\rho_{\mathbb{D}}\left(h^{-1}(a+t), \varphi_{t}\left(h^{-1}(a+n t)\right)\right)}{n}=\lim _{n \rightarrow \infty} \frac{\rho_{\Omega}(a+t, a+n t+t)}{n}$.

By Proposition 3.6, $\rho_{\Omega}(a+t, a+n t+t) \leq \rho_{\Theta}(a+t, a+n t+t)$. Therefore,

$$
\begin{aligned}
-\log \varphi_{t}^{\prime}(b) & \leq \lim _{n \rightarrow \infty} \frac{\rho_{\Theta}(a+t, a+(n+1) t)}{n} \\
& =\lim _{n \rightarrow \infty} \frac{\rho_{\mathbb{D}}\left(\Psi^{-1}(a+t), \Psi^{-1}(a+(n+1) t)\right)}{n} \\
& =\lim _{n \rightarrow \infty} \frac{1}{n} \rho_{\mathbb{D}}\left(\frac{1+i \sin (i \pi t / \lambda)}{1-i \sin (i \pi t / \lambda)}, \frac{1+i \sin (i \pi t(n+1) / \lambda)}{1-i \sin (i \pi t(n+1) / \lambda)}\right) \\
& =\lim _{n \rightarrow \infty} \frac{1}{n} \rho_{\mathbb{D}}\left(\frac{2+e^{-\pi t / \lambda}-e^{\pi t / \lambda}}{2-e^{-\pi t / \lambda}+e^{\pi t / \lambda}}, \frac{2+e^{-\pi t(n+1) / \lambda}-e^{\pi t(n+1) / \lambda}}{2-e^{-\pi t(n+1) / \lambda}+e^{\pi t(n+1) / \lambda}}\right) \\
& =\lim _{n \rightarrow \infty} \frac{1}{n} \log \left(\frac{e^{\pi t(n+1) / \lambda}-e^{-\pi t(n+1) / \lambda}}{e^{\pi t / \lambda}-e^{-\pi t / \lambda}}\right)=\frac{\pi t}{\lambda} .
\end{aligned}
$$

That is, $\varphi_{t}^{\prime}(b) \geq \exp (-\pi t / \lambda)$.

Proof of Proposition 2.3. Notice that $\gamma(s)=h^{-1}(h(\gamma(0))+s)$ for all $s>T$. By [Shapiro 1993, p. 162], for almost every $\varsigma \in \partial \mathbb{D}$ the (possibly infinite) radial limit $h^{*}(\varsigma)=\lim _{r \rightarrow 1} h(r \varsigma)$ exists and it is not a.e. constant on any subarc of $\partial \mathbb{D}$.

If $T>-\infty$, we know that the $\alpha$-limit $\alpha(\gamma)$ is a nonempty, compact, connected subset of $\partial \mathbb{D}$. We want to show that it is a single point. If this were not the case, $\alpha(\gamma)$ would be a nontrivial subarc of $\partial \mathbb{D}$. Hence, for each $a \in \alpha(\gamma)$ apart from the endpoints, the radius from 0 to $a$ would intersect $\gamma$ infinitely often. Thus there would exist $s_{n} \searrow T$ such that $\gamma\left(s_{n}\right)=r_{n} a$. The existence of the radial limit $h^{*}(a)=\lim _{r \rightarrow 1} h(r a)$ implies

$$
h^{*}(a)=\lim _{n} h\left(r_{n} a\right)=\lim _{n} h\left(\gamma\left(s_{n}\right)\right)=\lim _{n}\left(h(z)+s_{n}\right)=h(z)+T .
$$


So $h^{*}$ would be a.e. constant on $\alpha(\gamma)$, a contradiction. Therefore, $\alpha(\gamma)$ must be a single point; that is, the limit $a:=\lim _{s \rightarrow T} \gamma(s) \in \partial \mathbb{D}$ exists.

Now we have to show that, given $t>0, a$ is not a fixed point. Notice that $\lim _{s \rightarrow T^{+}} \varphi_{t}(\gamma(s))=\lim _{s \rightarrow T^{+}} h^{-1}(h(z)+s+t)=h^{-1}(h(z)+T+t) \in \mathbb{D}$. So, by Lindelöf's Theorem, $\lim _{r \rightarrow 1} \varphi_{t}(r a)=h^{-1}(h(z)+T+t) \in \mathbb{D}$. In particular, $\lim _{r \rightarrow 1} \varphi_{t}(r a) \neq a$ and $a$ cannot be a fixed point. Clearly, $h(z)+T \in \partial \Omega$.

If $T=-\infty$, the existence of the limit $a:=\lim _{s \rightarrow-\infty} \gamma(s) \in \partial \mathbb{D}$ is guaranteed by [Shapiro 1993, p. 162] and we showed in Lemma 4.2 that $a$ is a fixed point.

Recall that the invariant set of $\Omega$ is

$$
V(\Omega)=\operatorname{int}\left(\bigcap_{t \geq 0}(\Omega+t)\right),
$$

and that its connected components are denoted by $V_{j}(\Omega)$, for $j \in J$. We now summarize the basic properties of the invariant set and its connected components.

Lemma 5.1. (1) If $s \in \mathbb{R}$, then $V(\Omega)+s=V(\Omega)$.

(2) $V_{j}(\Omega)+s=V_{j}(\Omega)$ for all $s \in \mathbb{R}$ and all $j \in J$. In particular, each $V_{j}(\Omega)$ is a strip or a half-plane. Therefore, in the first case, there exist unique real numbers $a_{j}$ and $\beta_{j}(\Omega)$ such that

$$
V_{j}(\Omega)=\left\{z \in \mathbb{C}: a_{j}-\frac{1}{2} \beta_{j}(\Omega)<\operatorname{Im} z<a_{j}+\frac{1}{2} \beta_{j}(\Omega)\right\},
$$

and, in the second case, there is a real number $a_{j}$ such that

$$
V_{j}(\Omega)=\left\{z \in \mathbb{C}: a_{j}<\operatorname{Im} z\right\} \quad \text { or } \quad V_{j}(\Omega)=\left\{z \in \mathbb{C}: a_{j}>\operatorname{Im} z\right\} .
$$

Proof. (1) First suppose that $s>0$. Then

$$
\left(\bigcap_{t \geq 0}(\Omega+t)\right)+s=\bigcap_{t \geq 0}(\Omega+t+s)=\bigcap_{t \geq s}(\Omega+t)=\bigcap_{t \geq 0}(\Omega+t) .
$$

Therefore,

$$
V(\Omega)+s=\operatorname{int}\left(\left(\bigcap_{t \geq 0}(\Omega+t)\right)+s\right)=V(\Omega) .
$$

Now, if $s<0$, we have $V(\Omega)-s=V(\Omega)$. So, $V(\Omega)+s=V(\Omega)$.

(2) By the first part of this lemma, we have $V_{j}(\Omega)+s \subseteq V(\Omega)+s=V(\Omega)$. Moreover, $V_{j}(\Omega)+s$ is connected. So, there is $k$ such that $V_{j}(\Omega)+s \subseteq V_{k}(\Omega)$. Similarly, there is $l$ such that $V_{k}(\Omega)-s \subseteq V_{l}(\Omega)$. Therefore,

$$
V_{j}(\Omega)+s \subseteq V_{k}(\Omega) \subseteq V_{l}(\Omega)+s .
$$

So, $V_{j}(\Omega)=V_{l}(\Omega)$ and $V_{j}(\Omega)+s=V_{k}(\Omega)$. Why is $k=j$ ? Fix $z \in V_{j}(\Omega)$ and consider the curve $\tau \in[0, s] \rightarrow \sigma(\tau)=z+\tau \in V(\Omega)$. Clearly, $\sigma([0, s])$ is an arc 
in $V(\Omega)$ (and so it is included in one connected component) that begins in $V_{j}(\Omega)$ and ends in $V_{k}(\Omega)$. Therefore $V_{j}(\Omega)=V_{k}(\Omega)$.

Proof of Theorem 2.4. We notice that $\gamma_{z}$ is defined for all $t \in(-\infty, 0]$ if and only if $h(z)+\mathbb{R} \subset \Omega$.

(1) Take $z$ such that $h(z)+\mathbb{R}$ is in a half-plane $V_{j}(\Omega)$ and consider the curves $\Gamma_{1}(s)=h(z)-s$ and $\Gamma_{2}(s)=h(z)+s$ for $s>0$. We know, by the Denjoy-Wolff Theorem, that $\lim _{s \rightarrow \infty} h^{-1}\left(\Gamma_{2}(s)\right)=b$. By Lemma 4.1, we have

$$
\lim _{s \rightarrow \infty} h^{-1}\left(\Gamma_{1}(s)\right)=\lim _{s \rightarrow \infty} h^{-1}\left(\Gamma_{2}(s)\right)=b .
$$

(2) Let $z$ be a point of $\mathbb{D}$ such that $\Lambda:=h(z)+\mathbb{R} \subset \Omega$. Since, by assumption, $\lim _{s \rightarrow-\infty} h^{-1}(h(z)+s)=b$, the union $\gamma_{z} \cup\{b\}$ is a Jordan curve contained in $\mathbb{D} \cup\{b\}$ and satisfying $\varphi_{t}\left(\gamma_{z}\right)=\gamma_{z}$ for all $t \geq 0$. The curve $\gamma_{z}$ divides the unit disk into two connected components $\Theta_{1}$ and $\Theta_{2}$, where $\partial \Theta_{1}=\gamma_{z} \cup\{b\}$ and $\partial \Theta_{2}=$ $\partial \mathbb{D} \cup \gamma_{z}$. Transferring this situation to $\Omega$ we have $\Lambda=\partial h\left(\Theta_{1}\right) \cap \Omega$. Therefore, $\Lambda \subset \partial\left(h\left(\Theta_{1}\right)+t\right) \cap \Omega$ for all $t \geq 0$. That is, $\gamma_{z} \subset \partial \varphi_{t}\left(\Theta_{1}\right)$ for all $t \geq 0$. On the other hand, the restriction of $\varphi_{t}$ to $\bar{\Theta}_{1}$ (with the obvious extension to the point $b$ ) is a homeomorphism. So, $\varphi_{t}\left(\Theta_{1}\right)$ is a Jordan domain whose boundary contains the set $\gamma_{z}$. Therefore, $\varphi_{t}\left(\Theta_{1}\right)=\Theta_{1}$. Again, going over to $\Omega$, we have $h\left(\Theta_{1}\right)+t=h\left(\Theta_{1}\right)$ for all positive $t$, and a posteriori, for all $t \in \mathbb{R}$. That is, $h\left(\Theta_{1}\right)$ is a strip or a half-plane and it is contained in $V$.

Suppose that $h\left(\Theta_{1}\right)$ is a strip bounded by two lines. One of them is $\Lambda$. Let $\Upsilon$ denote the other line of the boundary of $h\left(\Theta_{1}\right)$. There exist $\beta>0$ and $a \in \mathbb{R} i$ such that the map

$$
\sigma(w)=\frac{1-e^{w / \beta} e^{-a / \beta}}{1+e^{w / \beta} e^{-a / \beta}}
$$

is a bijection from $h\left(\Theta_{1}\right)$ onto the unit disc. Notice that $\sigma$ has a continuous extension $\hat{\sigma}$ to $\overline{h\left(\Theta_{1}\right)}$ (with image $\overline{\mathbb{D}} \backslash\{-1,1\}$ ). By the Carathéodory Theorem (see, for example, [Milnor 1999, Theorem 17.16]) and since $\Theta_{1}$ is a Jordan domain, we have $\sigma \circ h_{\mid \Theta_{1}}$. can be extended to a homeomorphism from $\bar{\Theta}_{1}$ onto $\overline{\mathbb{D}}$. Given $w \in \Upsilon$ and taking a sequence $\left(w_{n}\right)$ in $h\left(\Theta_{1}\right)$ that converges to $w$, it is not difficult to prove that $\left(h^{-1}\left(w_{n}\right)\right)$ converges to $b$. So, $\sigma \circ h_{\mid \Theta_{1}}\left(h^{-1}\left(w_{n}\right)\right)$ converges to $\sigma \circ h_{\mid \Theta_{1}}(b) \in \partial \mathbb{D}$. On the other hand, $\sigma \circ h_{\mid \Theta_{1}}\left(h^{-1}\left(w_{n}\right)\right)=\left(\sigma\left(w_{n}\right)\right)$ converges to $\hat{\sigma}(w)$. That is, $\hat{\sigma}$ is constant on $\Upsilon$. This is a contradiction, since $w \mapsto e^{w / \beta}$ is not constant on any horizontal line. Therefore, $h\left(\Theta_{1}\right)$ is a half-plane contained in $V(\Omega)$ and we conclude that there is a half-plane $V_{j}(\Omega)$ such that $h\left(\Theta_{1}\right) \subset V_{j}(\Omega)$. In particular, $h(z) \in \overline{h\left(\Theta_{1}\right)} \subset \overline{V_{j}(\Omega)}$.

Proof of Theorem 2.5. (1) When we have a strip $V_{j}(\Omega)$ and two half-lines in $V_{j}(\Omega)$, say $h\left(z_{1}\right)+(-\infty, 0]$ and $h\left(z_{2}\right)+(-\infty, 0]$, we can apply Lemma 4.1 (joining the points $h\left(z_{1}\right)$ and $h\left(z_{2}\right)$ by a segment) to get $\lim _{s \rightarrow-\infty} h^{-1}\left(h\left(z_{1}\right)+s\right)=$ 
$\lim _{s \rightarrow-\infty} h^{-1}\left(h\left(z_{2}\right)+s\right)$. So, the fixed point $\xi\left(V_{j}(\Omega)\right)$ associated to $V_{j}(\Omega)$ is well-defined.

We now check that $\xi\left(V_{j}(\Omega)\right)$ is repulsive. We have to verify that

$$
1<\varphi_{t}^{\prime}\left(\xi\left(V_{j}(\Omega)\right)\right)<+\infty .
$$

Take $z_{n}=h^{-1}\left(a_{j} i-n\right)$. Then

$$
\rho_{\mathbb{D}}\left(z_{n}, \varphi_{t}\left(z_{n}\right)\right)=\rho_{\Omega}\left(a_{j} i-n, a_{j} i-n+t\right) \leq 2 \int_{\left[a_{j} i-n, a_{j} i-n+t\right]} \frac{|d w|}{\delta_{\Omega}(w)} \leq \frac{4 t}{\beta_{j}(\Omega)} .
$$

Therefore, there is a constant $\sigma<1$ such that the pseudo-hyperbolic distance between $z_{n}$ and $\varphi_{t}\left(z_{n}\right)$ is uniformly bounded by $\sigma$. That is,

$$
\left|\frac{z_{n}-\varphi_{t}\left(z_{n}\right)}{1-\bar{z}_{n} \varphi_{t}\left(z_{n}\right)}\right| \leq \sigma \quad \text { for all } n \text {. }
$$

Moreover, for $n$ large enough we have $\left|z_{n}\right| \geq \sigma$. From this, and using [Shapiro 1993, Exercise 1, p. 73], we conclude that

$$
\frac{1-\left|\varphi_{t}\left(z_{n}\right)\right|}{1-\left|z_{n}\right|} \leq \frac{1+\sigma}{1-\sigma}
$$

Hence,

$$
\liminf _{z \rightarrow \xi\left(V_{j}(\Omega)\right)} \frac{1-\left|\varphi_{t}(z)\right|}{1-|z|} \leq \frac{1+\sigma}{1-\sigma} .
$$

The Julia-Carathéodory Theorem implies that $\varphi_{t}^{\prime}\left(\xi\left(V_{j}(\Omega)\right)\right) \leq(1+\sigma) /(1-\sigma)<$ $+\infty$. To prove that $1<\varphi_{t}^{\prime}\left(\xi\left(V_{j}(\Omega)\right)\right)$, we will show that $\xi\left(V_{j}(\Omega)\right)$ is not the Denjoy-Wolff point of the semigroup $\left(\varphi_{t}\right)$. If this were the case, we would have $\alpha\left(h^{-1}(\Gamma)\right)=b$, where $\Gamma=\left\{a_{j} i\right\}+\mathbb{R}$. By Theorem $2.4, \Gamma$ would lie in the closure of one of the half-planes of $V(\Omega)$ : a contradiction because $\Gamma$ is in one of the strips of $V(\Omega)$.

(2) We begin by showing that the map $\xi$ that sends a strip to its associated fixed point is injective. Consider two components $V_{j}(\Omega)$ and $V_{l}(\Omega)$ such that $\xi\left(V_{j}(\Omega)\right)=$ $\xi\left(V_{l}(\Omega)\right)=\xi$ and assume that $V_{j}(\Omega) \neq V_{l}(\Omega)$. Take $\Gamma_{j}=\left\{a_{j} i\right\}+\mathbb{R}$ and $\Gamma_{l}=$ $\left\{a_{l} i\right\}+\mathbb{R}$. Set $\gamma_{j}=h^{-1}\left(\Gamma_{j}\right)$ and $\gamma_{l}=h^{-1}\left(\Gamma_{l}\right)$. The curves $\gamma_{j}$ and $\gamma_{l}$ are disjoint in $\mathbb{D}$ and connect $\xi$ to the Denjoy-Wolff point $b$; thus they bound a simply connected region $\Theta \subset \mathbb{D}$ such that $\partial \Theta=\gamma_{j} \cup \gamma_{l} \cup\{b, \xi\}$. Fix $t>0$. We have $\varphi_{t}\left(\gamma_{j}\right)=\gamma_{j}$ and $\varphi_{t}\left(\gamma_{l}\right)=\gamma_{l}$. Going over to $\Omega$ we have $\Gamma_{j} \cup \Gamma_{l} \subset \partial(h(\Theta)+t) \cap \Omega$; that is, $\gamma_{j} \cup \gamma_{l} \subset \partial \varphi_{t}(\Theta)$. On the other hand, the restriction of $\varphi_{t}$ to $\bar{\Theta}$ (with the obvious extensions to the points $b$ and $\xi$ ) is a homeomorphism. So, $\varphi_{t}(\Theta)$ is a Jordan domain whose boundary contains the Jordan curve $\gamma_{j} \cup \gamma_{l} \cup\{b, \xi\}$. Therefore, $\varphi_{t}(\Theta)=\Theta$. In particular, $h(\Theta)$ lies in $V(\Omega)$ and is included in a connected component of $V$, which is a contradiction since $h(\Theta) \cap V_{j}(\Omega) \neq \varnothing$ and $h(\Theta) \cap V_{l}(\Omega) \neq \varnothing$. Therefore, the map $\xi$ is injective. 
Now, we will see that the map $\xi$ is onto. Let $\xi \in \partial \mathbb{D}$ be a fixed point of $\varphi_{t}$ with $1<\varphi_{t}^{\prime}(\xi)<\infty$. Take $\varepsilon$ as the positive constant associated to the fixed point $\xi$ given in Lemma 4.4.

First, we are going to show that there are a strip $V_{j}(\Omega)$ and $0<r_{0}<1$ such that

$$
\bigcup_{r \geq r_{0}} B(h(r \xi), \varepsilon) \subset V_{j}(\Omega) .
$$

Let $\Lambda=h([0,1) \xi)$ and $\Lambda_{n}=\Lambda+n$. The curve $\Lambda$ connects $h(0)$ to $\infty$. Moreover, there are two constants $M_{1}$ and $M_{2}$ such that $M_{1} \leq \operatorname{Im} h(r \xi) \leq M_{2}$ for all $r$ and $\lim _{r \rightarrow 1^{-}} \operatorname{Re} h(r \xi)=-\infty$ (otherwise, by Lemma 4.1, $\xi$ would be the DenjoyWolff point). So, for each $n$, there is $\eta_{n}=h\left(r_{n} \xi\right)+n \in \Lambda_{n}$ such that $\operatorname{Re} \eta_{n}=-1$. Let $\left(\eta_{n_{k}}\right)$ be a subsequence of $\left(\eta_{n}\right)$ converging to a number $\eta$, with $\operatorname{Re} \eta=-1$, $M_{1} \leq \operatorname{Im} \eta \leq M_{2}$ and $\left|\eta_{n_{k}}-\eta\right|<\varepsilon / 2$ for all $k$. The choice of $\varepsilon$ implies that

$$
\delta_{\Omega}(h(r \xi)+n) \geq \delta_{\Omega}(h(r \xi)) \geq \varepsilon
$$

for all $r$. In particular,

$$
B(\eta, \varepsilon / 2) \subseteq B\left(\eta_{n_{k}}, \varepsilon\right)=B\left(h\left(r_{n_{k}} \xi\right), \varepsilon\right)+n_{k} \subset \Omega+n_{k}
$$

for all $k$. Therefore, $B(\eta, \varepsilon / 2) \subset V(\Omega)$. Let $V_{j}(\Omega)$ be the connected component of $V(\Omega)$ containing $B(\eta, \varepsilon / 2)$. Notice that $\eta_{n_{k}} \in B(\eta, \varepsilon / 2) \subset V_{j}(\Omega)$. Suppose that there is no $r_{0}$ such that

$$
\bigcup_{r \geq r_{0}} B(h(r \xi), \varepsilon) \subset V_{j}(\Omega) .
$$

Then, for infinitely many $k$, we can find $z_{k}$ with $\inf \left\{\left|z_{k}-w\right|: w \in \Lambda_{n_{k}}\right\}<\varepsilon / 2$, $\operatorname{Re} z_{k}<-1$ and $z_{k} \in \partial V_{j}(\Omega)$. Take $w_{k}=h\left(s_{k} \xi\right)+n_{k} \in \Lambda_{n_{k}}$ such that $\left|w_{k}-z_{k}\right|<\varepsilon / 2$. We have

$$
B\left(z_{k}, \varepsilon / 2\right) \subseteq B\left(w_{k}, \varepsilon\right)=B\left(h\left(s_{k} \xi\right), \varepsilon\right)+n_{k} \subset \Omega+n_{k}
$$

for all $k$. Now choose $m_{k}$ such that $\tilde{z}_{k}=z_{k}+m_{k}$ satisfies $-2 \leq \operatorname{Re}\left(\tilde{z}_{k}\right) \leq-1$ and, passing to a subsequence (still written the same), we consider that $\left(\tilde{z}_{k}\right)$ converges to a point $\zeta$. Of course, we can also suppose that $\left|\tilde{z}_{k}-\zeta\right|<\varepsilon / 4$ for all $k$, so

$$
B(\zeta, \varepsilon / 4) \subseteq B\left(\tilde{z}_{k}, \varepsilon / 2\right) \subset B\left(h\left(s_{k} \xi\right), \varepsilon\right)+n_{k}+m_{k} \subset \Omega+n_{k}+m_{k}
$$

for all $k$. Therefore, $B(\zeta, \varepsilon / 4) \subset V(\Omega)$. Let $V_{l}(\Omega)$ be the connected component of $V(\Omega)$ containing $B(\zeta, \varepsilon / 4)$. Then $\tilde{z}_{k} \in V_{l}(\Omega)$, which contradicts the fact that $\tilde{z}_{k}$ is in $\partial V_{j}(\Omega)$. That is,

$$
\bigcup_{r \geq r_{0}} B(h(r \xi), \varepsilon) \subset V_{j}(\Omega)
$$

for $r_{0}$ sufficiently close to 1 .

Next, we show that $V_{j}(\Omega)$ is a strip. Suppose $V_{j}(\Omega)$ is a half-plane, say $V_{j}(\Omega)=$ $\left\{z \in \mathbb{C}: a_{j}<\operatorname{Im} z\right\}$, and take $\Gamma_{1}(s)=\left(a_{j}+1\right) i-s$ and $\Gamma_{2}(s)=\left(a_{j}+1\right) i+s$ for 
$s>0$. Then, by Lemma 4.1, $\lim _{s \rightarrow \infty} h^{-1}\left(\Gamma_{1}(s)\right)=\lim _{s \rightarrow \infty} h^{-1}\left(\Gamma_{2}(s)\right)=b$, which contradicts the inequality $1<\varphi_{t}^{\prime}(\xi)$ (recall that $b$ is the Denjoy-Wolff point of $\varphi_{t}$ ).

Now we prove that $\xi\left(V_{j}(\Omega)\right)=\xi$. Take $\Gamma_{1}(s)=a_{j} i-s$ for $s>0$ and $\Gamma_{2}(r)=$ $h(r \xi)$ for $r<1$. We know that

$$
\lim _{s \rightarrow \infty} h^{-1}\left(\Gamma_{1}(s)\right)=\xi\left(V_{j}(\Omega)\right) \quad \text { and } \quad \lim _{r \rightarrow 1} h^{-1}\left(\Gamma_{2}(r)\right)=\xi .
$$

We check that the limits coincide. If there is a sequence $\left(w_{n}\right)$ in $\Gamma_{1}[0, \infty) \cap \Gamma_{2}[0,1)$ such that $w_{n} \rightarrow \infty$, clearly $\lim _{s \rightarrow \infty} h^{-1}\left(\Gamma_{1}(s)\right)=\lim _{r \rightarrow 1} h^{-1}\left(\Gamma_{2}(r)\right)$. Otherwise, using Lemma 4.1 again and bearing in mind that $\Gamma_{1}$ and $\Gamma_{2}(r)$ belong to $V_{j}(\Omega)$ for $r$ large enough, we deduce that $\lim _{s \rightarrow \infty} h^{-1}\left(\Gamma_{1}(s)\right)=\lim _{r \rightarrow 1} h^{-1}\left(\Gamma_{2}(r)\right)$.

The following lemma is well-known.

Lemma 5.2. Given $\xi \in \partial \mathbb{D}$ and a sequence $\left(z_{n}\right)$ in $\mathbb{D}$ that converges to $\xi$, the following assertions are equivalent.

(1) There exists $\alpha=\lim _{n} \operatorname{Arg}\left(1-\bar{\xi} z_{n}\right)$.

(2) There exists $m=\lim _{n} \frac{1-\bar{\xi} z_{n}}{\left|1-\bar{\xi} z_{n}\right|}$.

(3) There exists $\mu=\lim _{n} \frac{\operatorname{Im} \bar{\xi} z_{n}}{1-\operatorname{Re} \bar{\xi} z_{n}}$.

Moreover, if these assertions are satisfied, $e^{i \alpha}=m$ and $\mu=-\tan \alpha$.

Lemma 5.3. Suppose given $\xi \in \partial \mathbb{D}$, a repulsive boundary fixed point of the semigroup of analytic functions $\left(\varphi_{t}\right)$ with associated strip $V_{j}(\Omega)$, and $z \in \mathbb{D}$ with $h(z) \in V_{j}(\Omega)$. Then $\operatorname{Slope}^{-}\left(\gamma_{z}\right)$ is a single point of $\left(-\frac{\pi}{2}, \frac{\pi}{2}\right)$.

Proof. Given $s>t>0$, by the Invariant Schwarz-Pick Lemma [Shapiro 1993, p. 60] applied to the function $\varphi_{s-t}$, we have

$$
\left|\frac{\varphi_{s+1}(w)-\varphi_{s}(w)}{1-\overline{\varphi_{s+1}(w)} \varphi_{s}(w)}\right| \leq\left|\frac{\varphi_{t+1}(w)-\varphi_{t}(w)}{1-\overline{\varphi_{t+1}(w)} \varphi_{t}(w)}\right|
$$

for all $w \in \mathbb{D}$. In particular, taking the point $w=h^{-1}(h(z)-s-t$ ) (which is well-defined because $h(z) \in V_{j}(\Omega)$ ) we obtain

$$
\left|\frac{h^{-1}(h(z)-t+1)-h^{-1}(h(z)-t)}{1-\overline{h^{-1}(h(z)-t+1)} h^{-1}(h(z)-t)}\right| \leq\left|\frac{h^{-1}(h(z)-s+1)-h^{-1}(h(z)-s)}{1-\overline{h^{-1}(h(z)-s+1)} h^{-1}(h(z)-s)}\right| .
$$

That is, the function $f$ defined by

$$
f(t)=\left|\frac{h^{-1}(h(z)-t+1)-h^{-1}(h(z)-t)}{1-\overline{h^{-1}(h(z)-t+1)} h^{-1}(h(z)-t)}\right|=\left|\frac{\bar{\xi} h^{-1}(h(z)-t+1)-\bar{\xi} h^{-1}(h(z)-t)}{1-\bar{\xi} h^{-1}(h(z)-t+1) \bar{\xi} h^{-1}(h(z)-t)}\right|
$$


is increasing. Let $l=\lim _{t \rightarrow \infty} f(t)$. Since

$$
\frac{h^{-1}(h(z)-t+1)-h^{-1}(h(z)-t)}{1-\overline{h^{-1}(h(z)-t+1)} h^{-1}(h(z)-t)} \in \mathbb{D},
$$

for all $t$, we have $l \leq 1$.

Consider the curve

$$
\gamma(t)=\frac{\operatorname{Im} \bar{\xi} h^{-1}(h(z)-t)}{1-\operatorname{Re} \bar{\xi} h^{-1}(h(z)-t)},
$$

with $t>0$, and take a sequence $\left(t_{n}\right)$ increasing to infinity such that $\gamma\left(t_{n}\right)$ goes to $\mu$. By Proposition 4.5, the sequence $\left(h^{-1}\left(h(z)-t_{n}\right)\right)$ converges nontangentially to the point $\xi$. So, by Lemma 5.2, there exists $\alpha=\lim _{n} \operatorname{Arg}\left(1-\bar{\xi} h^{-1}\left(h(z)-t_{n}\right)\right) \in$ $\left(-\frac{\pi}{2}, \frac{\pi}{2}\right)$ and $\mu=-\tan \alpha \in \mathbb{R}$. Set $u_{n}=\operatorname{Re} \bar{\xi} h^{-1}\left(h(z)-t_{n}\right), v_{n}=\operatorname{Im} \bar{\xi} h^{-1}\left(h(z)-t_{n}\right)$, $a_{n}=\operatorname{Re} \bar{\xi} h^{-1}\left(h(z)-t_{n}+1\right)$, and $b_{n}=\operatorname{Im} \bar{\xi} h^{-1}\left(h(z)-t_{n}+1\right)$. We know that $v_{n} /\left(1-u_{n}\right)$ converges to $\mu$. Notice that $u_{n} \rightarrow 1$ and $v_{n} \rightarrow 0$. Moreover,

$$
\lim _{t \rightarrow \infty} \frac{1-\bar{\xi} \varphi_{1}\left(h^{-1}\left(h(z)-t_{n}\right)\right)}{1-\bar{\xi} h^{-1}\left(h(z)-t_{n}\right)}=\varphi_{1}^{\prime}(\xi) .
$$

In particular,

$$
\lim _{n} \frac{1-\left(a_{n}+i b_{n}\right)}{1-\left(u_{n}+i v_{n}\right)}=\varphi_{1}^{\prime}(\xi)>1 .
$$

A brief calculation shows that $\lim _{n}\left(1-a_{n}\right) /\left(1-u_{n}\right)=\varphi_{1}^{\prime}(\xi)$ and $\lim _{n} b_{n} /\left(1-u_{n}\right)=$ $\mu \varphi_{1}^{\prime}(\xi)$. On the other hand, using the definition of $f(t)$, we have

$$
\left|1-\left(a_{n}-i b_{n}\right)\left(u_{n}+i v_{n}\right)\right| f\left(t_{n}\right)=\left|\left(a_{n}+i b_{n}\right)-\left(u_{n}+i v_{n}\right)\right| .
$$

Dividing by $1-u_{n}$, we get

$$
\begin{aligned}
&\left|1+u_{n} \frac{1-a_{n}}{1-u_{n}}-\frac{b_{n}}{1-u_{n}} v_{n}-\frac{v_{n}}{1-u_{n}} a_{n} i+\frac{b_{n}}{1-u_{n}} u_{n} i\right| f\left(t_{n}\right) \\
&=\left|1-\frac{1-a_{n}}{1-u_{n}}+\frac{b_{n}}{1-u_{n}} i-\frac{v_{n}}{1-u_{n}} i\right| .
\end{aligned}
$$

Taking limits, we have $\left|1+\varphi_{1}^{\prime}(\xi)-\mu i+\mu \varphi_{1}^{\prime}(\xi) i\right| l=\left|1-\varphi_{1}^{\prime}(\xi)+\mu \varphi_{1}^{\prime}(\xi) i-\mu i\right|$, that is,

$$
\left|\frac{1+\varphi_{1}^{\prime}(\xi)}{1-\varphi_{1}^{\prime}(\xi)}-\mu i\right| l=|1-\mu i|
$$

Therefore,

$$
\mu^{2}\left(1-l^{2}\right)=\left(\frac{1+\varphi_{1}^{\prime}(\xi)}{1-\varphi_{1}^{\prime}(\xi)}\right)^{2} l^{2}-1
$$

Since the fraction in parentheses lies in $(-\infty,-1)$, we see that $l \neq 1$ and $\mu$ has at most two values. Therefore, by Lemma 5.2, the set $\operatorname{Slope}^{-}\left(\gamma_{z}\right)$ has at most two 
points, and being an interval, it must be a single point, which must be the number $\alpha$ found above. So, $\operatorname{Slope}^{-}\left(\gamma_{z}\right)$ is a single point of $\left(-\frac{\pi}{2}, \frac{\pi}{2}\right)$.

Proof of Theorem 2.6. Fix $t>0$. By Proposition 3.3, we have

$$
\begin{aligned}
\log \varphi_{t}^{\prime}(\xi) & =\lim _{r \rightarrow 1} \rho_{\mathbb{D}}\left(r \xi, \varphi_{t}(r \xi)\right) \\
& =\min _{\theta \in\left(-\frac{\pi}{2}, \frac{\pi}{2}\right)}\left\{\lim _{n} \rho_{\mathbb{D}}\left(z_{n}, \varphi_{t}\left(z_{n}\right)\right): z_{n} \rightarrow \xi, \lim _{n} \operatorname{Arg}\left(1-\bar{\xi} z_{n}\right)=\theta\right\} .
\end{aligned}
$$

By [Poggi-Corradini 2000, Corollary 1.5], given $\theta \in\left(-\frac{\pi}{2}, \frac{\pi}{2}\right)$, there is a sequence $\left(z_{n}\right)$ in $\mathbb{D}$ such that $z_{n}=\varphi_{t}\left(z_{n+1}\right), z_{n} \rightarrow \xi$ and $\operatorname{Arg}\left(1-\bar{\xi} z_{n}\right) \rightarrow \theta$. By Theorem 2.5, given such a sequence, there is a point $c \in \overline{V_{j}(\Omega)}$ such that $z_{n}=h^{-1}(c-n t)$. We check that $c \in V_{j}(\Omega)$. If this were not so, clearly $\lim _{s \rightarrow+\infty} \delta_{\Omega}(c-s)=0$, leading to a contradiction, since on the one hand

$$
\begin{aligned}
\rho_{\mathbb{D}}\left(z_{n}, \varphi_{t}\left(z_{n}\right)\right) & =\rho_{\Omega}(c-n t, c-n t+t) \\
& \geq \frac{1}{2} \log \left(1+\frac{t}{\min \left\{\delta_{\Omega}(c-n t), \delta_{\Omega}(c-n t+t)\right\}}\right),
\end{aligned}
$$

which tends to $\infty$, while on the other, by Proposition 3.3 and since $\theta \in\left(-\frac{\pi}{2}, \frac{\pi}{2}\right)$, we have

$$
\lim _{n \rightarrow \infty} \rho_{\mathbb{D}}\left(z_{n}, \varphi_{t}\left(z_{n}\right)\right)=\log \frac{\left|\varphi_{t}^{\prime}(\xi)+e^{-2 i \theta}\right|+\left|\varphi_{t}^{\prime}(\xi)-1\right|}{\left|\varphi_{t}^{\prime}(\xi)+e^{-2 i \theta}\right|-\left|\varphi_{t}^{\prime}(\xi)-1\right|}<\infty
$$

Conversely, given $c \in V_{j}(\Omega)$ and taking $z_{n}=h^{-1}(c-n t)$ for all $n$, by Lemma 5.3 , there is $\theta \in\left(-\frac{\pi}{2}, \frac{\pi}{2}\right)$ such that $\lim _{n} \operatorname{Arg}\left(1-\bar{\xi} z_{n_{k}}\right)=\theta$. Therefore,

$$
\begin{aligned}
\log \left(\varphi_{t}^{\prime}(\xi)\right) & =\min _{\theta \in\left(-\frac{\pi}{2}, \frac{\pi}{2}\right)}\left\{\lim _{n} \rho_{\mathbb{D}}\left(z_{n}, \varphi_{t}\left(z_{n}\right)\right): z_{n} \rightarrow \xi, \lim _{n} \operatorname{Arg}\left(1-\bar{\xi} z_{n}\right)=\theta\right\} \\
& =\min _{c \in V_{j}(\Omega)}\left\{\lim _{n} \rho_{\mathbb{D}}\left(z_{n}, \varphi_{t}\left(z_{n}\right)\right): z_{n}=h^{-1}(c-n t)\right\} .
\end{aligned}
$$

Thus, to calculate $\log \varphi_{t}^{\prime}(\xi)$, we will take $c \in V_{j}(\Omega)$, evaluate $\lim _{n} \rho_{\mathbb{D}}\left(z_{n}, \varphi_{t}\left(z_{n}\right)\right)$, where $z_{n}=h^{-1}(c-n t)$, and take the minimum of that limit. So fix an arbitrary $c$ in $V_{j}(\Omega)$. For all $n \in \mathbb{N}$, set $p_{n}=h^{-1}(c-n t)$ and let $\alpha_{p_{n}}$ be an automorphism of the unit disc such that $\alpha_{p_{n}}(0)=p_{n}$. Consider the function $f_{n}(z)=h \circ \alpha_{p_{n}}(z)+n t$. Then $f_{n}(0)=c$ and $f_{n}(\mathbb{D})=h(\mathbb{D})+n t=\Omega+n t \subseteq \Omega$, for all $n$. Thus $\left\{f_{n}: n \in \mathbb{N}\right\}$ is a normal family, and so has a subsequence $f_{n_{k}}$ converging uniformly on compact subsets of $\mathbb{D}$ to an analytic function $f$ on $\mathbb{D}$. Also, $f_{n+1}(\mathbb{D}) \subset f_{n}(\mathbb{D})$ for all $n$, so $f(\mathbb{D}) \subset \cap_{n} f_{n}(\mathbb{D})=\cap_{n}(\Omega+n t) \subset V(\Omega)$. Since $f(0)=c$, we have $f(\mathbb{D}) \subseteq V_{j}(\Omega)$. Moreover, $f_{n_{k}}^{-1}: V_{j}(\Omega) \rightarrow \mathbb{D}$ is also a normal family. So, it has a subsequence (still written the same) that converges uniformly on compact subsets of $V_{j}(\Omega)$ to an analytic function $g: V_{j}(\Omega) \rightarrow \overline{\mathbb{D}}$. Since $g(c)=0$, we have $g\left(V_{j}(\Omega)\right) \subseteq \mathbb{D}$. If 
$z \in \mathbb{D}$ and $k_{0}$ is large enough, then

$$
\left\{f_{n_{k}}(z): k \geq k_{0}\right\} \cup\{f(z)\}
$$

is a compact subset of $V_{j}$. Thus $z=f_{n_{k}}^{-1}\left(f_{n_{k}}(z)\right) \rightarrow g(f(z))$; that is, $z=g(f(z))$. Similarly, $w=f(g(w))$ for all $w \in V_{j}(\Omega)$. Thus, $f$ is one-to-one, $f(\mathbb{D})=V_{j}(\Omega)$, $f^{-1}=g$, and $f_{n_{k}}^{-1}$ converges uniformly on compact subsets of $V_{j}(\Omega)$ to $f^{-1}$. A simple calculation shows that

$$
f_{n}^{-1}(w)=\alpha_{p_{n}}^{-1}\left(h^{-1}(w-n t)\right)
$$

for all $w$. So, given $z \in \mathbb{D}$,

$$
\begin{aligned}
f_{n}^{-1}\left(f_{n}(z)+t\right) & =\alpha_{p_{n}}^{-1}\left(h^{-1}\left(f_{n}(z)+t-n t\right)\right) \\
& =\alpha_{p_{n}}^{-1}\left(h^{-1}\left(h \circ \alpha_{p_{n}}(z)+t\right)\right)=\alpha_{p_{n}}^{-1} \circ \varphi_{t} \circ \alpha_{p_{n}}(z) .
\end{aligned}
$$

In particular, taking $z=0$, we have $\alpha_{p_{n}}\left(f_{n}^{-1}(c+t)\right)=\varphi_{t}\left(p_{n}\right)$.

Recall that

$$
\Psi(z)=\frac{\beta_{j}}{\pi} \log \frac{1-z}{1+z}+\left(\operatorname{Re} c+a_{j} i\right)
$$

is another Riemann map of $V_{j}(\Omega)$. Thus, there is $\theta \in[0,2 \pi]$ and $d \in \mathbb{D}$ such that $f^{-1} \circ \Psi(z)=e^{i \theta}(z-d) /(1-\bar{d} z)$ for all $z \in \mathbb{D}$. Since $\Psi(d)=f(0)=c$, we get

$$
d=\frac{1-e^{\left(\pi / \beta_{j}(\Omega)\right) \operatorname{Im} c i} e^{-\left(\pi / \beta_{j}(\Omega)\right) a_{j} i}}{1+e^{\left(\pi / \beta_{j}(\Omega)\right) \operatorname{Im} c i} e^{-\left(\pi / \beta_{j}(\Omega)\right) a_{j} i}} .
$$

Now set $u=e^{\left(\pi / \beta_{j}(\Omega)\right) \operatorname{Im} c i} e^{-\left(\pi / \beta_{j}(\Omega)\right) a_{j} i}$ and $v=e^{\left(\pi / \beta_{j}(\Omega)\right) t}$. We obtain

$$
\begin{aligned}
\lim _{n \rightarrow \infty} \rho_{\mathbb{D}}\left(p_{n_{k}}, \varphi_{t}\left(p_{n_{k}}\right)\right) & \\
& =\lim _{n \rightarrow \infty} \rho_{\mathbb{D}}\left(\alpha_{p_{n_{k}}}(0), \alpha_{p_{n_{k}}}\left(f_{n_{k}}^{-1}(c+t)\right)\right) \\
& =\lim _{n \rightarrow \infty} \rho_{\mathbb{D}}\left(0, f_{n_{k}}^{-1}(c+t)\right)=\rho_{\mathbb{D}}\left(0, f^{-1}(c+t)\right) \\
& =\rho_{\mathbb{D}}\left(0, e^{i \theta} \frac{\Psi^{-1}(c+t)-d}{1-\bar{d} \Psi^{-1}(c+t)}\right)=\rho_{\mathbb{D}}\left(0, \frac{\Psi^{-1}(c+t)-d}{1-\bar{d} \Psi^{-1}(c+t)}\right) \\
& =\rho_{\mathbb{D}}\left(0, \frac{\frac{1-u v}{1+u v}-\frac{1-u}{1+u}}{1-\frac{u-1}{u+1} \frac{1-u v}{1+u v}}\right)=\rho_{\mathbb{D}}\left(0, u \frac{1-v}{1+u^{2} v}\right)=\rho_{\mathbb{D}}\left(0, \frac{1-v}{1+u^{2} v}\right) .
\end{aligned}
$$

Bearing in mind that

$$
d\left(0, \frac{1-v}{1+u^{2} v}\right)=\left|\frac{1-v}{1+u^{2} v}\right|=\frac{v-1}{\left|1+u^{2} v\right|}=\frac{v-1}{\sqrt{1+v^{2}+2 v \cos \left(2 \pi\left(\operatorname{Im} c-a_{j}\right) / \beta_{j}(\Omega)\right)}}
$$


and that $v$ does not depend on $c$, we have

$$
\begin{aligned}
& \log \varphi_{t}^{\prime}(\xi)=\min _{c \in V_{j}(\Omega)}\left\{\lim _{n} \rho_{\mathbb{D}}\left(z_{n}, \varphi_{t}\left(z_{n}\right)\right): z_{n}=h^{-1}(c-n t)\right\} \\
& =\min _{c \in V_{j}(\Omega)} \rho_{\mathbb{D}}\left(0, \frac{1-v}{1+u^{2} v}\right)=\log \frac{1+\min _{c \in V_{j}(\Omega)} d\left(0, \frac{1-v}{1+u^{2} v}\right)}{1-\min _{c \in V_{j}(\Omega)} d\left(0, \frac{1-v}{1+u^{2} v}\right)} \\
& =\log \frac{1+\min _{c \in V_{j}(\Omega)} \frac{v-1}{\sqrt{1+v^{2}+2 v \cos \left(2 \pi\left(\operatorname{Im} c-a_{j}\right) / \beta_{j}(\Omega)\right)}}}{1-\min _{c \in V_{j}(\Omega)} \frac{v-1}{\sqrt{1+v^{2}+2 v \cos \left(2 \pi\left(\operatorname{Im} c-a_{j}\right) / \beta_{j}(\Omega)\right)}}} \\
& =\log \frac{1+\frac{v-1}{v+1}}{1-\frac{v-1}{v+1}}=\frac{\pi}{\beta_{j}(\Omega)} t .
\end{aligned}
$$

That is, $\varphi_{t}^{\prime}\left(\xi\left(V_{j}(\Omega)\right)\right)=\exp \left(\pi t / \beta_{j}(\Omega)\right)$.

Proof of Theorem 2.7. Take $z$ such that

$$
h(z) \in\left(\bigcap_{t \geq 0}(\Omega+t)\right) \backslash\left(\bigcup_{j \in J} \overline{V_{j}(\Omega)}\right) .
$$

Then, by Proposition 2.3, $\alpha\left(\gamma_{z}\right)$ is a single point $\xi$ which is a fixed point. By Theorems 2.4 and $2.5, \xi$ is neither attractive nor repulsive. So, it must be a superrepulsive boundary fixed point of $\Phi$.

Suppose that there is another point $\tilde{z}$ such that $\gamma_{\tilde{z}} \neq \gamma_{z}$ and $\alpha\left(\gamma_{\tilde{z}}\right)=\{\xi\}$. Then $\gamma_{\tilde{z}}$ and $\gamma_{z}$ are disjoint curves in $\mathbb{D}$ connecting $\xi$ to $b$. So, they bound a simply connected region $\Theta \subset \mathbb{D}$ such that $\partial \Theta=\gamma_{\tilde{z}} \cup \gamma_{z} \cup\{\xi, b\}$. Let us fix $t>0$. We have that $\varphi_{t}\left(\gamma_{\tilde{z}}\right)=\gamma_{\tilde{z}}$ and $\varphi_{t}\left(\gamma_{z}\right)=\gamma_{z}$. Passing to $\Omega$, we have $h\left(\gamma_{z}\right) \cup h\left(\gamma_{\tilde{z}}\right) \subset$ $\partial(h(\Theta)+t) \cap \Omega$; that is, $\gamma_{\tilde{z}} \cup \gamma_{z} \subset \partial \varphi_{t}(\Theta)$. On the other hand, the restriction of $\varphi_{t}$ to $\bar{\Theta}$ (with the obvious extensions to the points $\xi$ and $b$ ) is a homeomorphism. Thus $\varphi_{t}(\Theta)$ is a Jordan domain whose boundary contains the Jordan curve $\gamma_{\tilde{z}} \cup \gamma_{z} \cup$ $\{\xi, b\}$. Therefore, $\varphi_{t}(\Theta)=\Theta$. In particular, $h(\Theta)$ lies in $V(\Omega)$ and is included in a connected component of $V(\Omega)$, which is a contradiction since $h(z) \notin \bigcup_{j \in J} \overline{V_{j}(\Omega)}$.

Lemma 5.4. Suppose $\left(z_{n}\right)$ and $\left(w_{n}\right)$ are two sequences in the unit disk that converge to $\xi \in \partial \mathbb{D}$ and such that

(1) $\operatorname{Arg}\left(1-\bar{\xi} z_{n}\right) \rightarrow \alpha$ and $\operatorname{Arg}\left(1-\bar{\xi} w_{n}\right) \rightarrow \beta$ with $\alpha, \beta \in\left[-\frac{\pi}{2}, \frac{\pi}{2}\right]$ and

(2) $\rho_{\mathbb{D}}\left(z_{n}, w_{n}\right) \rightarrow 0$.

Then $\alpha=\beta$. 
Proof. We have $z_{n}=\left(1-r_{n} e^{i \theta_{n}}\right) \xi$ and $w_{n}=\left(1-s_{n} e^{i \xi_{n}}\right) \xi$ with $\theta_{n}, \xi_{n} \in\left(-\frac{\pi}{2}, \frac{\pi}{2}\right)$. Notice that $\left\{n \in \mathbb{N}: r_{n} \geq s_{n}\right\}$ or $\left\{n \in \mathbb{N}: s_{n} \geq r_{n}\right\}$ is infinite. Without loss of generality, we assume that $r_{n} \geq s_{n}$ for all $n$. Again, taking a subsequence (still written the same), we suppose that $s_{n} / r_{n} \rightarrow \lambda \in[0,1]$. By Proposition 3.2, we have

$$
\left|\frac{z_{n}-w_{n}}{1-\bar{z}_{n} w_{n}}\right| \rightarrow 0
$$

Moreover,

$$
\left|\frac{z_{n}-w_{n}}{1-\bar{z}_{n} w_{n}}\right|=\left|\frac{-r_{n} e^{i \theta_{n}}+s_{n} e^{i \xi_{n}}}{r_{n} e^{-i \theta_{n}}+s_{n} e^{i \xi_{n}}-r_{n} s_{n} e^{i\left(\xi_{n}-\theta_{n}\right)}}\right|=\left|\frac{\left(s_{n} / r_{n}\right) e^{i\left(\xi_{n}-\theta_{n}\right)}-1}{1+\left(s_{n} / r_{n}\right) e^{i\left(\xi_{n}+\theta_{n}\right)}-s_{n} e^{i \xi_{n}}}\right| .
$$

If $\alpha+\beta= \pm \pi$, then $\alpha=\beta$. Otherwise, $1+\left(s_{n} / r_{n}\right) e^{i\left(\xi_{n}+\theta_{n}\right)}-s_{n} e^{i \xi_{n}} \rightarrow 1+\lambda e^{i(\beta+\alpha)}$, which is nonzero. So,

$$
\left|\frac{\left(s_{n} / r_{n}\right) e^{i\left(\xi_{n}-\theta_{n}\right)}-1}{1+\left(s_{n} / r_{n}\right) e^{i\left(\xi_{n}+\theta_{n}\right)}-s_{n} e^{i \xi_{n}}}\right| \rightarrow\left|\frac{\lambda e^{i(\beta-\alpha)}-1}{1+\lambda e^{i(\beta+\alpha)}}\right|=0 .
$$

Therefore, we obtain that $1=\lambda e^{i(\beta-\alpha)}$ and we conclude that $\alpha=\beta$.

Proof of Theorem 2.8. The proof that given $z \in \mathbb{D}$, the set $\omega\left(\operatorname{Arg}\left(1-\bar{b} \varphi_{t}(z)\right)\right)$ is a single point is similar to that of Lemma 5.3. So, $\operatorname{Slope}^{+}\left(\gamma_{z}\right)$ is a single point. Once we have obtained this, by Lemma 4.3, we see that $\operatorname{Slope}^{+}\left(\gamma_{z}\right) \in\left(-\frac{\pi}{2}, \frac{\pi}{2}\right)$.

Now consider the map $z \mapsto k(z)=\lim _{t \rightarrow \infty} \operatorname{Arg}\left(1-\bar{b} \varphi_{t}(z)\right)$. Assume for the moment the following two claims (which we will prove below).

Claim 1. The function $k$ is continuous. Therefore, $k(\mathbb{D})$ is an interval.

Claim 2. If $k\left(z_{1}\right)=k\left(z_{2}\right)$, then $\operatorname{Im} h\left(z_{1}\right)=\operatorname{Im} h\left(z_{2}\right)$.

By Lemma 4.4, there is $\varepsilon>0$ such that $\delta_{\Omega}(h(r \xi)) \geq \varepsilon$ for all $r \in[0,1)$. Take the real number $a$ such that

$$
\Omega \subseteq \Theta:=\left\{z \in \mathbb{C}: a-\frac{1}{2} v(\Omega)<\operatorname{Im} z<a+\frac{1}{2} v(\Omega)\right\} .
$$

Fix $M>0$. Recall that the set $\left\{z \in \mathbb{D}: \rho_{\mathbb{D}}(z,(-1,1)) \leq M\right\}$ is a lens associated to a certain angle $0<\alpha<\frac{\pi}{2}$.

We show that there are points $z$ such that the corresponding trajectory $\gamma_{z}$ satisfy that $\lim _{t \rightarrow+\infty} \rho_{\mathbb{D}}\left(\gamma_{z}(t),(-1,1)\right)>M$. Choose $\varepsilon>\delta>0$ with $\log (1+(\varepsilon-\delta) / \delta)>$ $4 M$, take $z_{1}, z_{2} \in \mathbb{D}$ with $\operatorname{Im} h\left(z_{1}\right)>a+\frac{1}{2} \nu(\Omega)-\delta$ and $\operatorname{Im} h\left(z_{2}\right)<a-\frac{1}{2} \nu(\Omega)+\delta$, and take $t>0$. Then $\delta_{\Omega}\left(h\left(z_{i}\right)+t\right) \leq \delta$ for $i=1,2$. By the Distance Lemma (Proposition 3.5), we have 


$$
\begin{aligned}
\rho_{\mathbb{D}}\left(\varphi_{t}\left(z_{i}\right),(-1,1)\right) & \geq \inf _{0<r<1} \rho_{\Omega}\left(h\left(z_{i}\right)+t, h(r)\right) \\
& \geq \inf _{s>0} \frac{1}{2} \log \left(1+\frac{\left|h\left(z_{i}\right)+t-h(r)\right|}{\min \left\{\delta_{\Omega}\left(h\left(z_{i}\right)+t\right), \delta_{\Omega}(r)\right\}}\right) \\
& \geq \frac{1}{2} \log \left(1+\frac{\varepsilon-\delta}{\delta}\right) \geq 2 M .
\end{aligned}
$$

Therefore, $\left|\operatorname{Slope}^{+}\left(\gamma_{z_{i}}\right)\right|>\alpha$ for $i=1,2$. Moreover, $\operatorname{Slope}^{+}\left(\gamma_{z_{1}}\right)$ and $\operatorname{Slope}^{+}\left(\gamma_{z_{2}}\right)$ have different signs, for the following reason: let $c$ be a point of $\Omega$ such that there is a sequence $r_{n} \nearrow 1$ with $\operatorname{Im} h\left(r_{n} \xi\right) \rightarrow \operatorname{Im} c$. Then, by Lemma 5.4, $k(c)=$ Slope $^{+}\left(\gamma_{c}\right)=0$. Of course, we may assume that $\operatorname{Re} c=\operatorname{Re} h\left(z_{1}\right)=\operatorname{Re} h\left(z_{2}\right)$ and that $\left[h\left(z_{1}\right), h\left(z_{2}\right)\right] \subset \Omega$. By Claim 2, the map $s \in\left[\operatorname{Im} h\left(z_{2}\right), \operatorname{Im} h\left(z_{1}\right)\right] \mapsto$ $k\left(h^{-1}(\operatorname{Re} c+i s)\right)$ is monotone. Therefore, $k\left(z_{1}\right) \geq k(0) \geq k\left(z_{2}\right)$ (or vice versa). This and Claim 1 imply that $(-\alpha, \alpha) \subset k(\mathbb{D})$ for all $\alpha$. Thus $k(\mathbb{D})=\left(-\frac{\pi}{2}, \frac{\pi}{2}\right)$.

Proof of Claim 1. Take $z_{n} \rightarrow z$. We have to check that $k\left(z_{n}\right) \rightarrow k(z)$. Since $k$ is constant on each trajectory, we may assume that, for $n$ large enough, $\operatorname{Re} h\left(z_{n}\right)=$ $\operatorname{Re} h(z)$ and the segment $\left[h\left(z_{n}\right), h(z)\right]$ is in $\Omega$. We have $\rho_{\mathbb{D}}\left(\varphi_{t}\left(z_{n}\right), \varphi_{t}(z)\right)$

$$
\begin{aligned}
& =\rho_{\Omega}\left(h\left(z_{n}\right)+t, h(z)+t\right) \leq 2 \int_{\left[h\left(z_{n}\right)+t, h(z)+t\right]} \frac{d s(x)}{\delta_{\Omega}(x)} \\
& \leq 2 \frac{\left|h\left(z_{n}\right)+t-h(z)-t\right|}{\min \left\{\delta_{\Omega}\left(h\left(z_{n}\right)+t\right), \delta_{\Omega}(h(z)+t)\right\}} \leq 2 \frac{\left|h\left(z_{n}\right)-h(z)\right|}{\min \left\{\delta_{\Omega}\left(h\left(z_{n}\right)\right), \delta_{\Omega}(h(z))\right\}} .
\end{aligned}
$$

Since $h\left(z_{n}\right)$ converges to $h(z)$ and $\delta_{\Omega}\left(h\left(z_{n}\right)\right)$ converges to $\delta_{\Omega}(h(z))$, which is nonzero, we obtain that $\rho_{\mathbb{D}}\left(\varphi_{t}\left(z_{n}\right), \varphi_{t}(z)\right) \rightarrow 0$. Now, take $t_{n} \rightarrow+\infty$ such that $\varphi_{t_{n}}\left(z_{n}\right)=\left(1-r_{n} e^{i \theta_{n}}\right) b$ with $r_{n} \leq \frac{1}{n}$, and $\left|\theta_{n}-k\left(z_{n}\right)\right| \leq \frac{1}{n}$ for all $n$. Without loss of generality, we may assume that $k\left(z_{n}\right) \rightarrow \beta \in\left[-\frac{\pi}{2}, \frac{\pi}{2}\right]$. Now consider $\varphi_{t_{n}}(z)=\left(1-s_{n} e^{i \xi_{n}}\right) b$. It is clear that $s_{n} \rightarrow 0$ and $\xi_{n} \rightarrow k(z)$. By Lemma 5.4, we have $\beta=k(z)$. That is, $k\left(z_{n}\right) \rightarrow k(z)$.

Proof of Claim 2. Take two orbits $\left\{\varphi_{t}\left(z_{1}\right): t \geq 0\right\}$ and $\left\{\varphi_{t}\left(z_{2}\right): t \geq 0\right\}$ and points $a_{n}=\left(1-r_{n} e^{i \theta_{n}}\right) b$ on the orbit $\left\{\varphi_{t}\left(z_{1}\right): t \geq 0\right\}$ and $b_{n}=\left(1-r_{n} e^{i \xi_{n}}\right) b$ on the orbit $\left\{\varphi_{t}\left(z_{2}\right): t \geq 0\right\}$, such that $\theta_{n} \rightarrow \alpha, \xi_{n} \rightarrow \alpha, \alpha \in\left(-\frac{\pi}{2}, \frac{\pi}{2}\right)$, and $r_{n} \rightarrow 0$. Then

$$
\left|\frac{a_{n}-b_{n}}{1-\bar{a}_{n} b_{n}}\right|=\left|\frac{-e^{i \theta_{n}}+e^{i \xi_{n}}}{e^{-i \theta_{n}}+e^{i \xi_{n}}-r_{n} e^{i\left(\xi_{n}-\theta_{n}\right)}}\right| \underset{n \rightarrow \infty}{\rightarrow} 0 .
$$

That is, $\rho_{\mathbb{D}}\left(a_{n}, b_{n}\right)$ tends to 0 . Setting $h\left(a_{n}\right)=h\left(z_{1}\right)+t_{n}$ and $h\left(b_{n}\right)=h\left(z_{2}\right)+s_{n}$, we have

$$
\begin{aligned}
\rho_{\mathbb{D}}\left(a_{n}, b_{n}\right) & =\rho_{\Omega}\left(h\left(a_{n}\right), h\left(b_{n}\right)\right)=\rho_{\Omega}\left(h\left(z_{1}\right)+t_{n}, h\left(z_{2}\right)+s_{n}\right) \\
& \geq \frac{1}{2} \log \left(1+\frac{\left|h\left(z_{1}\right)+t_{n}-h\left(z_{2}\right)-s_{n}\right|}{\min \left\{\delta_{\Omega}\left(h\left(z_{1}\right)+t_{n}\right), \delta_{\Omega}\left(h\left(z_{2}\right)+s_{n}\right)\right\}}\right) .
\end{aligned}
$$


Moreover, $\min \left\{\delta_{\Omega}\left(h\left(z_{1}\right)+t_{n}\right), \delta_{\Omega}\left(h\left(z_{2}\right)+s_{n}\right)\right\}$ is bounded below, so the sequence $\left(\left|h\left(z_{1}\right)+t_{n}-h\left(z_{2}\right)-s_{n}\right|\right)$ must go to zero. But

$$
\left|\operatorname{Im}\left(h\left(z_{1}\right)-h\left(z_{2}\right)\right)\right| \leq\left|h\left(z_{1}\right)+t_{n}-h\left(z_{2}\right)-s_{n}\right| .
$$

Therefore, $\operatorname{Im} h\left(z_{1}\right)=\operatorname{Im} h\left(z_{2}\right)$, proving the claim and Theorem 2.8.

Proof of Theorem 2.9. (1) Take $z_{1}, z_{2} \in \mathbb{D}$ and $\theta \in \operatorname{Slope}^{+}\left(\gamma_{z_{1}}\right)$. Then there is $\left(t_{n}\right) \nearrow \infty$ such that $\operatorname{Arg}\left(1-\bar{b} h^{-1}\left(h\left(z_{1}\right)+t_{n}\right)\right) \rightarrow \theta$. Bearing in mind Lemma 5.4, to obtain that $\theta \in \operatorname{Slope}^{+}\left(\gamma_{z_{2}}\right)$, it is enough to show that

$$
\lim _{n \rightarrow \infty} \rho_{\Omega}\left(h\left(z_{1}\right)+t_{n}, h\left(z_{2}\right)+\operatorname{Re}\left(h\left(z_{1}\right)-h\left(z_{2}\right)\right)+t_{n}\right)=0 .
$$

First notice we can assume that $\operatorname{Re} h\left(z_{1}\right)=\operatorname{Re} w$. We estimate the hyperbolic distance by integrating along the segment

$$
S=\left[h\left(z_{1}\right)+t_{n}, h\left(z_{2}\right)+\operatorname{Re}\left(h\left(z_{1}\right)-h\left(z_{2}\right)\right)+t_{n}\right],
$$

which is contained in $\Omega$ when $n$ is large enough:

$$
\begin{aligned}
\rho_{\Omega}\left(h\left(z_{1}\right)+t_{n}, h\left(z_{2}\right)+\operatorname{Re}\left(h\left(z_{1}\right)-h\left(z_{2}\right)\right)+t_{n}\right) & \leq 2 \int_{S} \frac{d s(z)}{\delta_{\Omega}(z)} \\
& \leq 2\left|h\left(z_{1}\right)-h\left(z_{2}\right)\right| \max _{z \in S} \frac{1}{\delta_{\Omega}(z)} .
\end{aligned}
$$

If we take $n$ large enough, we have

$$
\begin{aligned}
\max _{z \in S} & \frac{1}{\delta_{\Omega}(z)} \\
& =\frac{1}{\min \left\{\delta_{\Omega}\left(h\left(z_{1}\right)+t_{n}\right), \delta_{\Omega}\left(h\left(z_{2}\right)+\operatorname{Re}\left(h\left(z_{1}\right)-h\left(z_{2}\right)\right)+t_{n}\right)\right\}} \\
& \leq \frac{1}{\min \left\{\delta_{\Omega}\left(w+t_{n}\right)-\left|h\left(z_{1}\right)-w\right|, \delta_{\Omega}\left(w+t_{n}\right)-\left|h\left(z_{2}\right)+\operatorname{Re}\left(h\left(z_{1}\right)-h\left(z_{2}\right)\right)-w\right|\right\}} \\
& =\frac{1}{\delta_{\Omega}\left(w+t_{n}\right)-\max \left\{\left|h\left(z_{1}\right)-w\right|,\left|h\left(z_{2}\right)+\operatorname{Re}\left(h\left(z_{1}\right)-h\left(z_{2}\right)\right)-w\right|\right\}},
\end{aligned}
$$

which tends to 0 as $n$ goes to $\infty$. That is,

$$
\lim _{n \rightarrow \infty} \rho_{\Omega}\left(h\left(z_{1}\right)+t_{n}, h\left(z_{2}\right)+\operatorname{Re}\left(h\left(z_{1}\right)-h\left(z_{2}\right)\right)+t_{n}\right)=0 .
$$

(2) Since Slope ${ }^{+}\left(\gamma_{z}\right)$ is an interval, it is enough to apply Lemma 4.6.

Proof of Theorem 2.10. (1) Recall that $\operatorname{Slope}^{-}\left(\gamma_{z}\right)$ is a nonempty, compact and connected subset of $\left[-\frac{\pi}{2}, \frac{\pi}{2}\right]$. Moreover, it is clear that $\lim _{s \rightarrow-\infty} \delta_{\Omega}(h(z)-s)<\infty$. So, by Lemma 4.6, Slope $^{-}\left(\gamma_{z}\right)$ is equal to $-\frac{\pi}{2}$ or $\frac{\pi}{2}$. 
(2) Arguing as in the proof of Theorem 2.8, the function

$$
w \mapsto k(w)=\lim _{s \rightarrow \infty} \operatorname{Arg}\left(1-\bar{b} h^{-1}(w-s)\right)
$$

is continuous as $w$ runs over one of the half-planes of the invariant set. Since $k(w)$ is either $-\frac{\pi}{2}$ or $\frac{\pi}{2}$ for all $w$, it must be constant.

Proof of Theorem 2.11. (1) It is enough to apply Lemma 5.3 and [Poggi-Corradini 2000, Corollary 1.5].

(2) Recall that $\operatorname{Slope}^{-}\left(\gamma_{z}\right)$ is a nonempty, compact, connected subset of $\left[-\frac{\pi}{2}, \frac{\pi}{2}\right]$. Moreover, it is clear that $\lim _{s \rightarrow+\infty} \delta_{\Omega}(h(z)-s)=0$. On the one hand,

$$
\begin{aligned}
\rho_{\mathbb{D}}\left(h^{-1}(h(z)-s), \varphi_{t}\left(h^{-1}(h(z)\right.\right. & -s))) \\
& =\rho_{\Omega}(h(z)-s, h(z)-s+t) \\
& \geq \frac{1}{2} \log \left(1+\frac{t}{\min \left\{\delta_{\Omega}(h(z)-s), \delta_{\Omega}(h(z)-s+t)\right\}}\right),
\end{aligned}
$$

which tends to $\infty$ with $s$. On the other hand, if there is $\left(s_{n}\right) \nearrow \infty$ such that

$$
\lim _{n \rightarrow \infty} \operatorname{Arg}\left(1-\overline{\xi\left(V_{j}(\Omega)\right)} h^{-1}\left(h(z)-s_{n}\right)\right)=\theta \in\left(-\frac{\pi}{2}, \frac{\pi}{2}\right),
$$

by Proposition 3.3, we have

$$
\begin{aligned}
\lim _{n \rightarrow \infty} \rho_{\mathbb{D}}\left(h^{-1}\left(h(z)-s_{n}\right)\right. & \left., \varphi_{t}\left(h^{-1}\left(h(z)-s_{n}\right)\right)\right) \\
& =\log \frac{\left|\varphi_{t}^{\prime}\left(\xi\left(V_{j}(\Omega)\right)\right)+e^{-2 i \theta}\right|+\left|\varphi_{t}^{\prime}\left(\xi\left(V_{j}(\Omega)\right)\right)-1\right|}{\left|\varphi_{t}^{\prime}\left(\xi\left(V_{j}(\Omega)\right)\right)+e^{-2 i \theta}\right|-\left|\varphi_{t}^{\prime}\left(\xi\left(V_{j}(\Omega)\right)\right)-1\right|}<\infty,
\end{aligned}
$$

yielding a contradiction. That is, $\operatorname{Slope}^{-}\left(\gamma_{z}\right)$ is either $\frac{\pi}{2}$ or $\frac{\pi}{2}$.

\section{Acknowledgement}

The works of Poggi-Corradini [1998; 2000] on discrete iteration of analytic selfmaps of the unit disk have been an important source of inspiration for the mathematical techniques developed in this paper.

\section{References}

[Berkson and Porta 1978] E. Berkson and H. Porta, "Semigroups of analytic functions and composition operators", Michigan Math. J. 25:1 (1978), 101-115. MR 58 \#1112 Zbl 0382.47017

[Cowen 1981] C. C. Cowen, "Iteration and the solution of functional equations for functions analytic in the unit disk", Trans. Amer. Math. Soc. 265:1 (1981), 69-95. MR 82i:30036 Zbl 0476.30017

[Cowen and MacCluer 1995] C. C. Cowen and B. D. MacCluer, Composition operators on spaces of analytic functions, CRC Press, Boca Raton, FL, 1995. MR 97i:47056 Zbl 0873.47017 
[Cowen and Pommerenke 1982] C. C. Cowen and C. Pommerenke, "Inequalities for the angular derivative of an analytic function in the unit disk", J. London Math. Soc. (2) 26:2 (1982), 271-289. MR 84a:30006 Zbl 0476.30001

[Heins 1981] M. Heins, "Semigroups of holomorphic maps of a Riemann surface into itself which are homomorphs of the set of positive reals considered additively", pp. 314-331 in E. B. Christoffel: The influence of his works on mathematics and the physical sciences (Aachen/Monschau, 1979), edited by P. L. Butzer and F. Feher, Birkhäuser, Basel, 1981. MR 84f:30012 Zbl 0474.30022

[Milnor 1999] J. Milnor, Dynamics in one complex variable: introductory lectures, Vieweg, Braunschweig, 1999. MR 2002i:37057 Zbl 0946.30013

[Needham and King 1994] D. J. Needham and A. C. King, "On meromorphic complex differential equations”, Dynam. Stability Systems 9:2 (1994), 99-122. MR 95m:34010 Zbl 0813.34005

[Poggi-Corradini 1998] P. Poggi-Corradini, "Angular derivatives at boundary fixed points for selfmaps of the disk”, Proc. Amer. Math. Soc. 126:6 (1998), 1697-1708. MR 98g:30049 Zbl 0891.30014

[Poggi-Corradini 2000] P. Poggi-Corradini, "Canonical conjugations at fixed points other than the Denjoy-Wolff point”, Ann. Acad. Sci. Fennica Math. 25:2 (2000), 487-499. MR 2001f:30033 Zbl 0958.30012

[Pommerenke 1975] C. Pommerenke, Univalent functions, Vandenhoeck \& Ruprecht, Göttingen, 1975. MR 58 \#22526 Zbl 0298.30014

[Pommerenke 1992] C. Pommerenke, Boundary behaviour of conformal maps, Grundlehren der Mathematischen Wissenschaften 299, Springer, Berlin, 1992. MR 95b:30008 Zbl 0762.30001

[Shapiro 1993] J. H. Shapiro, Composition operators and classical function theory, Springer, New York, 1993. MR 94k:47049 Zbl 0791.30033

[Shoikhet 2001] D. Shoikhet, Semigroups in geometrical function theory, Kluwer Academic Publishers, Dordrecht, 2001. MR 2002g:30012 Zbl 0980.30001

[Siskakis 1985] A. G. Siskakis, Semigroups of composition operators and the Cesàro operator on $H^{p}(D)$, Ph.D. thesis, University of Illinois, 1985.

Received March 31, 2004. Revised November 4, 2005.

\author{
MANUEl D. CONTRERAS \\ CAMino de los Descubrimientos, S/N \\ Departamento de MATEMÁtica Aplicada II \\ ESCUELA SUPERIOR DE INGENIEROS \\ UNIVERSIDAD DE SEVILLA \\ 41092, SEVILLA \\ SPAIN \\ contreras@esi.us.es \\ SANTIAGO DÍAZ-MADRIGAL \\ CAMINO DE LOS DESCUBRIMIENTOS, S/N \\ Departamento de Matemática Aplicada II \\ ESCUELA SUPERIOR DE INGENIEROS \\ UNIVERSIDAD DE SEVILLA \\ 41092, SEVILLA \\ SPAIN \\ madrigal@us.es
}

